\title{
Use of Causality Conditions in Quantum Theory
}

\author{
M. Gell-ManN, Institute of Nuclear Studies and Department of Physics, University of Chicago, Chicago, Illinois \\ M. L. Goldberger, * Princeton University, Princeton, New Jersey \\ AND \\ W. E. ThIrring, $†$ Institute for Advanced Study, Princeton, New Jersey
}

(Received May 24, 1954)

\begin{abstract}
The limitations on scattering amplitudes imposed by causality requirements are deduced from the demand that the commutator of field operators vanish if the operators are taken at points with space-like separations. The problems of the scattering of spin-zero particles by a force center and the scattering of photons by a quantized matter field are discussed. The causality requirements lead in a natural way to the well-known dispersion relation of Kramers and Kronig. A new sum rule for the nuclear photoeffect is derived and the scattering of photons by nucleons is discussed.
\end{abstract}

\section{INTRODUCTION}

$I^{\mathrm{N}}$ $\mathrm{N}$ the past few years there have been a number of papers which consider the question of deducing general conditions on scattering amplitudes on the basis of causality requirements. This has been a revival of the original work of Kronig ${ }^{1}$ and $\mathrm{Kramers}^{2}$ and followed for the most part a suggestion by Kronig ${ }^{3}$ that causality requirements should be added to the usual conditions on the $S$ matrix, namely, Lorentz invariance and unitarity. The actual requirements imposed by causality have been stated in various ways. Tiomno and Schutzer, ${ }^{4}$ discussing the scattering of nonrelativistic particles, impose the condition that the scattered wave should be zero before the incident wave hit the scatterer. This work has been criticised by van $\mathrm{Kampen}^{5}$ who discusses the same problem. He demands that the total probability outside the scatterer never exceed its initial value. In the case of photons, a different approach has been made. In several of the papers the problem is treated from the standpoint of the classical electrodynamics description of the propagation of light through a medium with an index of refraction where one imposes the condition that signals should not propagate faster than the velocity of light. This was, in fact, the original approach to Kronig and Kramers, and has been developed most rigorously by Toll. ${ }^{6}$ The connection between the index of refraction and the scattering amplitude is then made by means of the optical relation:

$$
n(\nu)=1+\left(2 \pi c^{2} / \nu^{2}\right) \rho F(\nu),
$$

* On leave of absence from the University of Chicago, Chicago, Illinois.

$\dagger$ Present address, Physikalisches Institute, Universitat Bern, Bern, Switzerland.

1 R. Kronig, J. Opt. Soc. Am. 12, 547 (1926).

${ }^{2}$ H. A. Kramers, Atti. congr. intern. fisici, Como, 2, 545 (1927).

${ }^{3}$ R. Kronig, Physica 12, 543 (1946).

4 W. Schutzer and J. Tiomno, Phys. Rev. 83, 249 (1951).

5 N. G. van Kampen, Phys. Rev. 91, 1267 (1953).

${ }^{6} \mathrm{~J}$. S. Toll, Princeton thesis (unpublished). In this paper, one finds complete references, and numerous very interesting applications of the dispersion relations for light. where $\rho$ is the density of scattering centers and $F(\nu)$ is the coherent forward scattering amplitude. This approach does not seem too satisfactory in that it is based on classical electrodynamics and further that the relationship to individual scatterers is established only in a circuitous way via Eq. (1.1). A more direct treatment has been given by van Kampen ${ }^{7}$ who considers the scattering of photons by a spherically symmetric scatterer outside of which the interaction is exactly zero. His treatment, however, is still based on classical electrodynamics. The causality condition in this paper is that of Tiomno and Schutzer.

With a single exception, no one has to our knowledge discussed the problem of relativistic particles with mass. The exception is the work of Moshinsky ${ }^{8}$ who has treated the special case of particles interacting with a zero-range force.

The causality requirement in the present paper is as follows: The quantum-mechanical formulation of the demand that waves do not propagate faster than the velocity of light is, as is well known, the condition that the measurements of two observable quantities should not interfere if the points of measurement are space-like to each other. In the case of the propagation of bosons to which we limit ourselves in this paper, our formal statement is that the commutator of two Heisenberg operators for the field in question shall vanish if the operators are taken at space-like points. This condition is sufficient to enable us to discuss the case of particles with mass, as well as photons. Our demand is a much more stringent one than that of Tiomno and Schutzer; not only can waves not be scattered before the incident wave arrives, but even after the arrival one must wait the appropriate time to receive a signal.

The principle object of the work of van Kampen and Schutzer and Tiomno was to obtain detailed information on the analytic behavior of the $S$ matrix in the complex energy plane. In these treatments, the behavior of phase shifts for individual angular momenta are

${ }^{7}$ N. G. van Kampen, Phys. Rev. 89, 1072 (1953).

8 M. Moshinsky (unpublished). We are indebted to Profess or A. S. Wightman for showing us Dr. Moshinsky's paper. 
studied. An entirely different and very powerful approach to this specific problem has been made by Wigner (unpublished lectures at Princeton, 1953) whose method unfortunately cannot be directly applied to the case of photons. In all of these treatments, it is essential to assume that the interaction vanishes exactly (or is completely known) outside some finite region in configuration space. This limitation has not been completely overcome in our work. In the case of the interaction between photons and a matter field we are forced to rely on the familiar field theoretic device of adiabatic switching on and off of interactions. We shall not discuss the implications of this procedure, but shall simply accept it, as is conventionally done, as one of the apparently necessary shortcomings of the present formalism.

In this paper we shall use our quantum-mechanical causality requirement, as described above, to discuss the scattering of spin-zero particles and of photons. We shall deduce results essentially equivalent to the Kramers-Kronig dispersion relations and which are independent of the size of a region of interaction. Wigner and van Kampen demonstrate that the scattering amplitudes for individual angular momenta are analytic in the upper half-plane, if regarded as functions of the energy. If one wants relations between real and imaginary parts of the amplitudes for real values of the energy (i.e., a dispersion relation), one needs not only analytically but also certain boundedness of the amplitudes. It turns out that one must supply an exponential factor depending on the size of the scattering region to secure the desired boundedness of the individual scattering amplitudes. This greatly complicates the dispersion relations and makes them essentially useless for our purposes. One finds, however, that certain combinations of the phase shifts, for example, the forward scattering amplitude, have boundedness properties of such a nature that any reference to the size of the region of interaction may be avoided. The dispersion relations deduced in connection with the forward amplitude are the classical ones of Kramers and Kronig and many applications have been made of them in the past. (See reference 6.) There are other combinations of phase shifts for which the size of the region plays no role; these are essentially the derivatives of the scattering amplitude evaluated in the forward direction. The boundedness conditions on these are more obscure and we have made no applications of them. Whereas all of the previous dispersion relations have been confined to completely coherent forward scattering amplitudes, our results allow a consideration of processes in which there are spin flips and polarization changes.

In Sec. II we shall discuss first the simple case of a spin-zero particle interacting with a spherically symmetric force center; the interaction is assumed to vanish exactly outside a finite radius. The possibility of bound states and particle absorption are included. The more realistic case of photons interacting with a quantized matter field is treated in Sec. III. We have used a perturbation theoretic approach to the problem, expanding the Heisenberg operators of the photon field in powers of the electric charge, $e$. The results obtained are in all probability rigorously true to all orders of $e$, but for our applications only the order $e^{2}$ was needed; it should not be difficult to generalize our result to all powers of $e^{2}$. In Sec. IV, the connection between the causality requirement on the forward amplitude deduced in Secs. III and IV and the dispersion relations is discussed. In all cases causality yields the information that the forward scattering amplitude has certain analyticity and boundedness properties and it is the mathematical consequences of these conditions that are explore in Sec. IV. In Sec. V we treat in detail the application of the dispersion relations to two cases of physical interest. The first is the deduction of a sum rule for photonuclear reactions in which the integrated photonuclear cross section (integrated up to the meson threshold) is related to the observed photomeson cross section. It is a generalization of the dipole sum rule given by Bethe and Levinger. ${ }^{9}$ The second application is to the problem of the scattering of $\gamma$ rays by protons. We use the dispersion relation to put limits on the forward scattering amplitude and in the case of low frequencies we get a limit on the phase shifts for the low multipoles. Since this case is of considerable experimental interest, we have gone beyond the results obtainable from our dispersion relations, mentioned above, to discuss the general features of $\gamma-p$ scattering on the basis of some additional assumptions. Finally, in Appendix B we collect the relevant formulas for a phase shift analysis of this process.

\section{SPIN-ZERO FIELD IN AN EXTERNAL POTENTIAL}

Note added in proof.-It has been pointed out to us by Professor N. G. van Kampen that in this section the arguments pertaining to particles with mass are lacking in rigor. In the following sections, however, we use only results pertaining to massless fields. See reference 12 .

In order to explain our procedure most simply, we shall consider first the rather academic case of a neutral spin-zero field interacting with a time-independent spherically symmetric external potential which vanishes outside a region of radius $a / 2$. We shall assume for the time being that there are no bound states and that there is no particle absorption and later indicate the manner in which the results are modified if these complications are included. In this simple problem we may imagine that the solutions to the field equations are obtained; in terms of these normal modes we may confine our attention to one-particle states. It is perhaps worth noting that our problem is quite similar to that treated by van Kampen; it is simpler in that we deal with a . scalar rather than a vector field (which difference is

${ }^{9}$ J. Levinger and H. A. Bethe, Phys. Rev. 78, 115 (1950). 
trivial) but more complicated because we consider a nonvanishing rest mass.

We now make use of the fact that the vacuum expectation value of the commutator of two field operators, $\phi(x)$ and $\phi\left(x^{\prime}\right)$, vanishes if the points $x, x^{\prime}$ are space-like to each other. (Obviously any matrix element of such a commutator vanishes under these circumstances since the commutator itself vanishes.) $\phi(x)$ is the Heisenberg operator of our scalar field. Since such a commutator vanishes outside the light cone and is zero for $t_{0}=t_{0}{ }^{\prime}$, it must be an odd function of time. We may therefore confine our attention to positive values of the time difference $t_{0}-t_{0}{ }^{\prime}$. We may then define a retarded Green's function, $G_{\text {ret }}\left(x, x^{\prime}\right)$, as follows :

$$
i\left\langle 0\left|\left[\phi(x), \phi\left(x^{\prime}\right)\right]\right| 0\right\rangle_{\eta}\left(x-x^{\prime}\right) \equiv G_{\mathrm{ret}}\left(x, x^{\prime}\right),
$$

where $\eta\left(x-x^{\prime}\right)$ is zero if $x_{0}^{\prime}>x_{0}$, and is unity of $x_{0}>x_{0}{ }^{\prime}$. The properties of $G_{\text {ret }}\left(x, x^{\prime}\right)$ which we shall exploit are

$$
\begin{aligned}
& G_{\text {ret }}\left(x, x^{\prime}\right)=0 \quad \text { if } \quad\left(x-x^{\prime}\right)^{2}>0 \\
& =0 \text { if } x_{0}<x_{0}^{\prime} .
\end{aligned}
$$

By hypothesis we need consider only one-particle states which we may choose to be continuum states corresponding to plane waves plus outgoing spherical waves. If such states be characterized by the momentum vector $\mathbf{k}$ of the incident plane wave, we may write

$$
\langle 0|\phi(x)| \mathbf{k}\rangle=(2 w)^{-\frac{1}{2}} U_{\mathbf{k}}(\mathbf{r}) e^{-i w x_{0}}
$$

where $w=+\left(k^{2}+\mu^{2}\right)^{\frac{1}{2}}$, with $\mu$ the particle $(h=c=1$ throughout this paper). We choose a unit quantization volume and normalize the $U_{\mathrm{k}}$ to plane waves of unit amplitude. The factor $1 /(2 w)^{\frac{1}{2}}$ insures that $G_{\text {ret }}\left(x, x^{\prime}\right)$ reduces to the conventional retarded Green's function for a free field.

We may now rewrite Eq. (2.1) in the form:

$$
\begin{aligned}
G_{\mathrm{ret}}\left(x, x^{\prime}\right)= & i \eta\left(x-x^{\prime}\right) \int \frac{d^{3} k}{(2 \pi)^{3}}\left\{\langle 0|\phi(x)| \mathbf{k}\rangle\left\langle\mathbf{k}\left|\phi\left(x^{\prime}\right)\right| 0\right\rangle\right. \\
& \left.-\left\langle 0\left|\phi\left(x^{\prime}\right)\right| \mathbf{k}\right\rangle\langle\mathbf{k}|\phi(x)| 0\rangle\right\} \\
= & i \eta\left(x-x^{\prime}\right) \int \frac{d^{3} k}{(2 \pi)^{3}} \frac{1}{2 w}\left\{U_{\mathbf{k}}(r) U_{\mathbf{k}}{ }^{*}(r)\right. \\
\times & \left.e^{-i w\left(x_{0}-x_{0}^{\prime}\right)}-U_{\mathbf{k}}\left(\mathbf{r}^{\prime}\right) U_{\mathbf{k}}^{*}(\mathbf{r}) e^{i w\left(x_{0}-x_{0}^{\prime}\right)}\right\} .
\end{aligned}
$$

Outside the region of interaction $\left(r, r^{\prime}>a / 2\right)$ we may write the eigenfunctions $U_{\mathbf{k}}(\mathbf{r})$ as

$$
\begin{aligned}
U_{\mathbf{k}}(\mathbf{r})= & \sum_{l=0}^{\infty} \frac{(2 l+1) i^{l}}{2 k r}\left[h_{l}^{(1)}(k r)+h_{l}^{(1)^{*}}(k r)\right] P_{l}(\mathbf{k}, \mathbf{r}) \\
& +\sum_{l=0}^{\infty} \frac{(2 l+1) i^{l}}{2 k r}\left[e^{2 i \delta l}-1\right] h_{l}^{(1)}(k r) P_{l}(\mathbf{k}, \mathbf{r})
\end{aligned}
$$

where $h_{l}^{(1)}(x)=(\pi x / 2)^{\frac{1}{2}} H_{l+\frac{1}{2}}^{(1)}(x)$ and $H_{l+\frac{1}{2}}^{(1)}(x)$ is the customary Hankel function. ${ }^{10}$ These functions are conveniently defined for our purposes as follows:

$$
\begin{aligned}
h_{l}^{(1)}(x) & =(-i) \exp [i(k r-\pi / 2)] q_{l}(x), \\
q_{l}(x) & =\sum_{s=0}^{l} \frac{i^{s}(l+s) !}{s !(l-s) !(2 x)^{s}} .
\end{aligned}
$$

The $\delta_{l}$ are the usual (real) phase shifts and the $P_{l}(\mathbf{k}, \mathbf{r})$ are Legendre polynomials of the angle between $\mathbf{k}$ and $\mathbf{r}$. If we substitute the eigenfunctions (2.5) into (2.4), the angular integrations are easily carried out with the aid of the spherical harmonic addition theorem. Setting $|\mathbf{r}|=\left|\mathbf{r}^{\prime}\right|$, and $x_{0}-x_{0}{ }^{\prime}=T$, we find for $G_{\text {ret }}\left(x, x^{\prime}\right)$ the expression:

$$
\begin{aligned}
& G_{\mathrm{ret}}\left(\mathbf{r}, \mathbf{r}^{\prime}, T\right)=\Delta_{\mathrm{ret}}\left(x-x^{\prime}\right)+\eta(T) \frac{\pi i}{r^{2}} \int_{0}^{\infty} \frac{d k}{2 w(2 \pi)^{3}} \\
& \begin{array}{r}
\times \sum_{l=0}^{\infty}(2 l+1)\left[\left(e^{2 i \delta l}-1\right)\left\{h_{l}^{(1)}(r)\right\}^{2}+\left(e^{-2 i \delta l}-1\right)\right. \\
\left.\times\left\{h_{l}^{(1)^{*}}(k r)\right\}^{2}\right] P_{l}\left(\mathbf{r}, \mathbf{r}^{\prime}\right)\left[e^{-i w t}-e^{i w t}\right],
\end{array}
\end{aligned}
$$

where $\Delta_{\text {ret }}(x)$ is defined as $-\eta(x) \Delta(x)$ with $\Delta(x)$ as given by Schwinger. ${ }^{11}$ We now define the scattering amplitude for individual angular momenta $f_{l}(k)$ by

$$
f_{l}(k)=\left(e^{2 i \delta_{l}(k)}-1\right) / 2 i k,
$$

and furthermore define them for negative values of $k$ as

$$
f_{l}(-k) \equiv f_{l}^{*}(k) \text {. }
$$

Then using the elementary relation,

we find

$$
h_{l}^{(1)}(-x)=(-1)^{l+1} h_{l}^{(1)^{*}}(x),
$$

$$
\begin{aligned}
& G_{\mathrm{ret}}\left(r, r^{\prime}, T\right)=\Delta_{\mathrm{ret}}\left(x-x^{\prime}\right)+ \frac{\eta(T)}{(2 \pi)^{2} r^{2}} \int_{0}^{\infty} \frac{k d k}{2 w} \\
& \times\left[\sum(2 l+1) f_{l}(k) q_{\imath}{ }^{2}\left(\frac{k R}{2}\right)(-1)^{l} P\left(\mathbf{r}, \mathbf{r}^{\prime}\right)\right] \\
& \times e^{i k R}\left(e^{-i w T}-e^{i w T}\right),
\end{aligned}
$$

where we have set $2 r=R$. The conditions to be imposed on $G_{\text {ret }}$, Eq. (2.2), are of course satisfied by $\Delta_{\text {ret }}\left(x-x^{\prime}\right)$; to insure their being satisfied by the entire function $G_{\text {ret }}\left(r, r^{\prime}, T\right)$ we must demand that the function $G(R, \vartheta T)$ defined by

$$
\begin{aligned}
G(R, \vartheta, T) & =\eta(T) \int_{-\infty}^{\infty} d k \frac{k}{2 w}\left[\sum_{l=0}^{\infty}(2 l+1) f_{l}(k)(-1)^{l}\right. \\
\times & \left.P_{l}(\cos \vartheta) q_{l}{ }^{2}\left(\frac{k R}{2}\right)\right] e^{i k R}\left(e^{-i w T}-e^{i w T}\right)
\end{aligned}
$$

${ }^{10}$ G. N. Watson, Bessel Functions (Cambridge University Press, New York, 1944), p. 73.

${ }^{11}$ J. Schwinger, Phys. Rev. 75, 651 (1949). 
(where $\vartheta$ is the angle between $\mathbf{r}, \mathbf{r}^{\prime}$ ), vanish for

$$
R \sin (\vartheta / 2)>T, \quad R>a .
$$

The restrictions on the $f_{l}(k)$ which are required to make $G$ vanish under the conditions (2.12) are exceedingly complicated and we have not studied the problem in this form. One obtains considerable simplifications by choosing $\vartheta=\pi$ which corresponds to putting $\mathbf{r}^{\prime}=-\mathbf{r}$. As we shall see below, the restriction to the forward direction may be relaxed somewhat and we can obtain information about scattering at other angles. Observing that $P_{l}(-1)=(-1)^{l}$ and writing $G(R, 0, T)$ simply $G(R, T)$ we find that (2.11) and (2.12) may be replaced by

$$
\begin{aligned}
& G(R, T)=\eta(T) \int_{\infty}^{\infty} d k \frac{k}{2 w} \sum_{l=0}^{\infty}(2 l+1) f_{l}(k) q_{l}{ }^{2}(k R / 2) \\
& \times e^{i k R}\left(e^{-i w T}-e^{i w T}\right) \\
&=0 \text { for } \quad R>T, \quad R>a .
\end{aligned}
$$

We shall drop the $\eta(T)$ factor and simply restrict our attention to positive values of $T$. It is convenient to rewrite (2.13) in the form

$$
\begin{aligned}
G(R, T) & =\int_{-\infty}^{\infty} d \nu \epsilon(\nu) \int_{-\infty}^{\infty} d k \cdot k \delta\left(\nu^{2}-w^{2}\right) \\
& =0, \quad R>T, \quad R>a,
\end{aligned}
$$

where

$$
M(k, R)=\sum_{l=0}^{\infty}(2 l+1) f_{l}(k) q_{l}{ }^{2}(k R / 2) .
$$

Using the $\delta$ function to carry out the integration over $k$, one finds, writing $k_{l}=+\left(\nu^{2}-\mu^{2}\right)^{\frac{1}{2}}$, that $G(R, T)$ may be written as

$$
\begin{aligned}
G(R, T)=\int_{\mu}^{\infty} & \frac{d \nu}{2} M\left(k_{\nu}, R\right) e^{+i k_{\nu} R-i \nu T} \\
& +\int_{\infty}^{\mu} \frac{d \nu}{2} M\left(-k_{\nu}, R\right) e^{-i k_{\nu} R-i \nu T} \\
& +\int_{-\infty}^{-\mu} \frac{d \nu}{2} M\left(-k_{\nu}, R\right) e^{-i k_{\nu} R-i \nu T} \\
& \quad+\int_{-\mu}^{\infty} \frac{d \nu}{2} M\left(k_{\nu}, R\right) e^{i k_{\nu} R-i \nu T} .
\end{aligned}
$$

Let us now consider the function $\left(\nu^{2}-\mu^{2}\right)^{\frac{1}{2}}$ in the complex $\nu$ plane, and introduce cuts from $-\infty$ to $-\mu$ and from $\mu$ to $\infty$. If we choose the branch of $\left(\nu^{2}-\mu^{2}\right)^{\frac{1}{2}}$ which is real and positive when $\nu>\mu$, we find that (2.16) may be written as

$$
\begin{aligned}
G(R, T)=\int_{C} \frac{d \nu}{2} M\left(\left(\nu^{2}-\mu^{2}\right)^{\frac{1}{2}}, R\right) \\
\quad \times \exp \left\{i\left[\left(\nu^{2}-\mu^{2}\right)^{\frac{1}{2}} R-\nu T\right]\right\},
\end{aligned}
$$

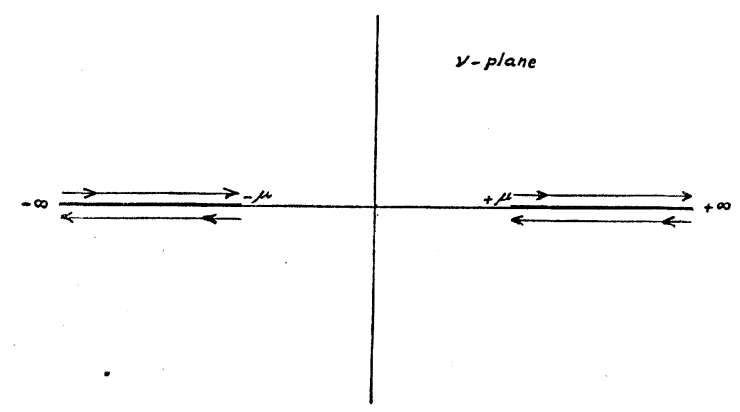

FIG. 1. The contour of integration $C$ for the integral in Eq. (2.16). The heavy lines running from $-\infty$ to $-\mu$ and from $\mu$ to $+\infty$ are the branch lines of the function $\left(\nu^{2}-\mu^{2}\right)^{3}$.

where $C$ is the contour shown in Fig. 1. In the strip between $-\mu$ and $+\mu,\left(\nu^{2}-\mu^{2}\right)^{\frac{1}{2}}=+i\left(\mu^{2}-\nu^{2}\right)^{\frac{1}{2}}$.

In place of the time interval $T$ we shall introduce the variable $z=R-T$ so that $G(R, T)$ becomes a function of $R$ and $z$; our requirement is that $G(R, z)$ shall vanish for $R>a, z>0$. There is every reason to believe that $G(R, z)$ is a well-behaved function of $z$ so long as $z$ is greater than zero; there is, however, a distinct possibility in many cases of physical interest that $G$ involve terms proportional to $\delta(z)$ and $\delta^{\prime}(z)$. This would in fact be the case if the total cross section were to approach a constant for large $\nu$. It is perhaps worth noting that these singularities are not unexpected; the original commutator is certainly singular on the light cone. In order to obtain a formula valid even for $z=0$, we first first multiply $G$ by $e^{-i q 1 z}$ and integrate over $z$ from $z$ to infinity (which cannot change the value since $G$ is zero over this range) giving $q_{1}$, a negative imaginary part to insure convergence, and then multiply by $e^{-i q_{2} z}$ and again integrate from $z$ to infinity. We obtain then

$$
\begin{array}{r}
H(R, z)=\int_{z}^{\infty} d z^{\prime} \int_{z^{\prime}}^{\infty} d z^{\prime \prime} e^{i\left(q_{\left.12 z^{\prime}+q z^{\prime \prime}\right)}\right) G\left(R, z^{\prime \prime}\right),} \\
H(R, z)=-e^{-i\left(q_{1}+q_{2}\right) z} \int_{C} \frac{d \nu}{2} \frac{M\left\{\left(\nu^{2}-\mu^{2}\right)^{\frac{1}{2}}, R\right\}}{\left(\nu-q_{1}\right)\left(\nu-q_{2}\right)} \\
\quad \times \exp \left\{i\left[\left(\nu^{2}-\mu^{2}\right)^{\frac{1}{2}}-\nu\right] R+i \nu Z\right\} .
\end{array}
$$

Strictly speaking, the upper limits of the $z$ integrations should be $R$, since $T>0$ and thus for fixed $R, z$ cannot exceed $R$. We shall be interested, however, only in the limit as $R \rightarrow \infty$, in which case, the contributions from the upper limits are negligible. The frequencies $q_{1}$ and $q_{2}$ will be chosen at our convenience. We note that the integrand in Eq. (2.18) is square integrable over the interval $-\infty$ to $+\infty$.

Consider now $G(R, z)$ for very large values of $R$. The contributions to the integral coming from portions of the contour for which $\left(\nu^{2}-\mu^{2}\right)^{\frac{1}{2}}$ and $\nu$ have the opposite algebraic sign will contain a rapidly varying exponential factor and make a vanishingly small contribution, $O(1 / R)$, in comparison with the portions for which 
$\left(\nu^{2}-\mu^{2}\right)^{\frac{1}{2}}$ and $\nu$ have the same sign. We may therefore discard the part of the contour below the real axis in Fig. 1, provided we retain only the lowest powers in $1 / R .^{12}$ Suppose now we were to augment our contour by the inclusion of the strip from $-\mu$ to $+\mu$, staying slightly above the real axis. From our choice of cuts, $\left(\nu^{2}-\mu^{2}\right)^{\frac{1}{2}}=i\left(\mu^{2}-\nu^{2}\right)^{\frac{1}{2}}$, and consequently we have a decreasing exponential dependence on $R$ as well as a rapidly varying oscillation from the factor $\exp (-i \nu R)$. Such contributions are clearly negligible in comparison with terms of order $1 / R$ or larger coming from the outer portions of the contour. We may therefore replace the integral over $C$ by one extending from $-\infty$ to $\infty$ on a line just above the real axis. Retaining only the leading term in powers of $1 / R$, we conclude that we must have

$$
\begin{aligned}
\int_{-\infty}^{\infty} d \nu \frac{\sum_{l=0}^{\infty}\left[(2 l+1) f_{l}\left\{\left(\nu^{2}-\mu^{2}\right)^{\frac{1}{2}}\right\}\right]}{\left(\nu-q_{1}\right)\left(\nu-q_{2}\right)} \\
\quad \times \exp \left\{i\left[\left(\nu^{2}-\mu^{2}\right)^{\frac{1}{2}}-\nu\right] R\right\} e^{i \nu z}=0, \quad z \geqslant 0 .
\end{aligned}
$$

We have, of course, assumed that $M(k, R)$ does not do anything spectacular on the strip $-\mu<\nu<\mu$ provided we stay slightly above the real axis. There may be singularities on the real axis and in fact if there are bound states there will be poles. This point will be discussed further below.

The sum in Eq. (2.19) is just the forward scattering amplitude $f(\nu)$ for a particle of momentum $k=\left(\nu^{2}-\mu^{2}\right)^{\frac{1}{2}}$. As is discussed in Sec. IV, we may conclude from (2.19) that the function $g(\nu, R)$ defined by

$$
g(\nu, R)=\frac{f(\nu)}{\left(\nu-q_{1}\right)\left(\nu-q_{2}\right)} \exp \left\{i\left[\left(\nu^{2}-\mu^{2}\right)^{\frac{1}{2}}-\nu\right] R\right\},
$$

must be analytic in the upper half of the $\nu$ plane. The discussion in Sec. IV also shows that $g(\nu, R)$ is bounded in the upper half-plane and we shall now show that this boundedness is not dependent on the exponential factor. To see this, put $\nu=i y$ and consider the exponential for very large positive values of $y$. The exponential becomes $\exp \left(-\mu^{2} R / 2 y\right)$ and thus tends to unity for $y \rightarrow \infty$. We may conclude therefore that the function $\bar{f}(\nu)$ given by

$$
\bar{f}(\nu)=f(\nu) /\left[\left(\nu-q_{1}\right)\left(\nu-q_{2}\right)\right]
$$

is analytic in the upper half-plane (and is bounded for all values of $\nu, 0<\arg \nu<\pi)$.

We must now generalize the above result to include also the possibilities of bound states and particle ab-

${ }^{12}$ E. T. Whittaker and G. N. Watson, Modern Analysis (Cambridge University Press, New York, 1948), p. 172. The theorems referred to, the Riemann-Lebesgue lemmas, do not exactly cover the case at hand. The exact conditions on $M\left[\left(\nu^{2}-\mu^{2}\right)^{\frac{1}{2}}, R\right]$ which must obtain for the above remarks to be true have not been studied. The claims made seem very plausible, and in the only case where applications are made $(\mu=0)$, the required extension of the Riemann-Lebesgue lemmas is trivial. See the discussion following Eq. (2.24). sorption. In case there are bound states, there are, as is well known, poles on the real $\nu$ axis from $-\mu$ to $\mu$. However, these do not seriously trouble us because we always integrate on a contour slightly above the real axis. The contributions of the small indentations of the contour (made to avoid the poles) are precisely cancelled by the inclusion of the bound states in the sum over intermediate states detailed in Eq. (2.4). In the limit, then, as $\nu$ approaches the real axis in an integral such as in Eq. (2.19), one takes the principal value at the poles of the forward scattering amplitude $f(\nu)$.

If there is absorption of particles, we must imagine augmenting our considerations to allow for the additional channel (or channels) describing the alternate modes. This requires formal modifications of a variety familiar in nuclear reaction theory; roughly speaking, we must increase our set of eigenfunctions to include not only those states initiated by a plane wave in the elastic scattering channel, but also to include those states initiated by plane waves in other channels (i.e., the reactions inverse to the absorption processes) which have only outgoing waves in the elastic channel. The outcome of these manipulations is that the previous conclusions drawn from the function $f(\nu)$ in Eq. (2.21) continue to be true provided that $f(\nu)$ be interpreted as the coherent elastic forward scattering amplitude. In this case the phase shifts $\delta_{l}$ are, of course, complex.

The previous considerations relating to particles with mass do not appear to have very interesting applications. Of greater interest is the case of zero rest mass to which we now turn. The real physical problem of photons interacting with a quantized matter field will be discussed in the next section, but for the time being we shall continue the discussion with the amplified model of spin-zero "photons" and an external potential. All of our previous considerations remain valid but, of course, become simpler. We shall rewrite some of the equations for the special case $\mu=0$.

The equation analogous to Eq. (2.16) becomes

$\begin{aligned} G(R, T)=\int_{-\infty}^{\infty} \frac{d \nu}{2} e^{-i \nu T} \epsilon(\nu) & \left\{M(|\nu|, R) e^{i|\nu| R}\right. \\ & \left.-M(-|\nu|, R) e^{-i|\nu| R}\right\}\end{aligned}$

which may be written in terms of $z=R-T$ as

$G(R, z)=\int_{-\infty}^{\infty} \frac{d \nu}{2} e^{i \nu z}\left\{M(\nu, R)-M(-\nu, R) e^{-2 i \nu R}\right\}$

The equation corresponding to (2.18) is obviously

$$
\begin{aligned}
H(R, z)=-e^{i\left(q_{1}+q_{2}\right) z} \int_{-\infty}^{\infty} \frac{d \nu}{2} \frac{e^{i \nu z}}{\left(\nu-q_{1}\right)\left(\nu-q_{2}\right)} \\
\times\left\{M(\nu, R)-M(-\nu, R) e^{-2 i \nu R}\right\} .
\end{aligned}
$$

The argument now proceeds essentially as before. If we look first at the leading term in the $1 / R$ expansion 
of $M(\nu, R)$ (which is independent of $1 / R$ ) we may conclude that the second term in Eq. (2.24) goes to zero in the limit $R \rightarrow \infty$. This may be seen by using the assumed square integrability of $M(-\nu, R) e^{i \nu z} /\left(\nu-q_{1}\right)$ $\times\left(\nu-q_{\sigma}\right)$ together with Parseval's theorem. Imposing the condition that $H(R, z)$ should vanish for $z>0$ leads to the conclusion that the forward scattering amplitude, $f(\nu)$, is analytic in the upper half-plane and that our previously defined function $\bar{f}(\nu)$ is bounded in the upper half-plane.

Thus far we have considered only the leading term in the $1 / R$ expansion of $M(\nu, R)$. This term is the forward scattering amplitude. One may well ask if anything can be said about directions other than forward. We have been unable to obtain any very simple relations, however some information may be found in the following way: If we had not made the the special choice of angle $\vartheta=\pi$ in Eq. (2.11), we would still have been able to carry through all the steps leading to Eqs. (2.18) and (2.24); the only difference that appears is that $M(\nu, R)$, say, in (2.24) becomes replaced by

$$
M(\nu, R, \cos \vartheta)=\sum_{l=0}^{\infty}(2 l+1) f_{l}(\nu) P_{l}(\cos \vartheta) q_{l}\left(\frac{\nu R}{2}\right),
$$

where we have replaced $\vartheta$ by $\pi-\vartheta$. Note that the leading term in the $1 / R$ expansion of $M(\nu, R, \cos \vartheta)$ is just the total scattering amplitude, $f(\vartheta)$. Our causality requirement takes the form

$$
\begin{array}{r}
H(R, z, \cos \vartheta)=- \\
\quad e^{-i\left(q_{1}+q_{2}\right) z} \int_{-\infty}^{\infty} \frac{d \nu}{2} \frac{e^{\prime \nu z}}{\left(\nu-q_{1}\right)\left(\nu-q_{2}\right)} \\
\quad \times\{M(\nu, R, \cos \vartheta) \\
\left.\quad-M(-\nu, R, \cos \vartheta) e^{-2 i \nu R}\right\} \\
=0, \quad \text { for } \quad R \cos (\vartheta / 2)>T, \quad R>a .
\end{array}
$$

We now differentiate $H$ with respect to $\cos \vartheta$ and put $\vartheta=0$; an attempt to continue as before to isolate the leading term in the $1 / R$ expansion of $\partial M / \partial \cos \vartheta$ leads to some difficulty in general. The reason is that $P_{l}^{\prime}(1)$ $=l(l+1) / 2$, so that $\partial M / \partial \cos \vartheta$ will probably be proportional to $\nu^{3}$ (for large $\nu$ ) if $f(\nu) \sim \nu$ as has previously been assumed. Before applying the Riemann-Lebesgue theorem, we must supply two more powers of $\nu$ in the denominator by using our integration technique. [See procedure leading to Eq. (2.18).] When these are supplied, we may conclude that the function $g(\nu)$ defined by

$$
g(\nu)=\frac{[\partial f(\nu, \cos \vartheta) / \partial \cos \vartheta]_{\cos \vartheta=1}}{\left(\nu-q_{1}\right)\left(\nu-q_{2}\right)\left(\nu-q_{3}\right)\left(\nu-q_{4}\right)}
$$

with the $q$ 's to be chosen conveniently, is analytic and bounded in the upper half $\nu$ plane. It is clear that there is an infinite sequence of such relations, obtained by carrying out the successive differentiations. In every case more powers of $\nu$ must be supplied in the denomi- nator to insure the proper boundedness. We have made no attempt to solve in any sense for the individual scattering amplitudes $f_{l}(\nu)$ from this set of relations.

\section{QUANTIZED ELECTROMAGNETIC AND MATTER FIELDS}

We pass now to a consideration of the more interesting situation where the quantized electromagnetic field interacts with a quantized matter field. We shall consider a system whose interaction with the radiation field may be described by an interaction Hamiltonian density $-j_{\mu}(x) A_{\mu}(x)$, where $j_{\mu}(x)$ is the current density four-vector operator of the matter system and $A_{\mu}(x)$ the usual four-vector potential of the radiation field. The procedure we follow at this point is strictly analogous to that used in the previous section. We demand that the commutator of two Heiseberg field operators $\mathbf{A}_{\lambda}(x), \mathbf{A}_{\sigma}(y)$ shall vanish for space-like separations of $x$ and $y$. It is convenient to consider a matrix element of the commutator between an initial state for which there are no photons and the matter field is a state $i$ and a final state with no photons and the matter system in a state $f$. We shall restrict our attention to such states $i$ and $f$ for which the energies $E_{i}$ and $E_{f}$ are equal. It is again convenient to restrict our attention to positive time intervals $x_{0}-y_{0}$ and to deal with the retarded function $\oint_{\lambda_{\sigma}}(x, y)$ defined by

$$
\mathcal{G}_{\lambda \sigma}(x, y)=i\left\langle 0, f\left|\left[\mathbf{A}_{\sigma}(x), \mathbf{A}_{\sigma}(y)\right]\right| i, 0\right\rangle_{\eta}(x-y) .
$$

Our demand is that $\mathcal{G}_{\lambda \sigma}(x, y)$ be zero for $(x-y)^{2}>0$. There is, of course, no way known at present to compute the Heisenberg operators A except by perturbation theory. Fortunately this is not too serious since the lowest order perturbation calculations in the radiation field are very accurate. We shall work to second order in the electric charge $e$. It is unnecessary then for us to carry out any renormalizations. We assume that all interactions other than with the radiation field are taken into account exactly and we work in such an interaction representation. (For example, if we consider the scattering of $\gamma$ rays by a hydrogen atom, we use the Furry interaction representation. $)^{13}$ To the second order in $e$, then, the Heisenberg operator $\mathbf{A}_{\lambda}(x)$ takes the well known form ${ }^{14}$

$$
\begin{aligned}
\mathbf{A}_{\lambda}(x)= & A_{\lambda}(x)+i \int_{-\infty}^{\infty} d^{4} x^{\prime}\left[H\left(x^{\prime}\right), A_{\lambda}\left(x^{\prime}\right)\right] \eta\left(x-x^{\prime}\right) \\
+i^{2} \int_{-\infty}^{\infty} d^{4} x^{\prime} \int_{-\infty}^{\infty} d^{4} x^{\prime \prime} & {\left[H\left(x^{\prime \prime}\right),\left[H\left(x^{\prime}\right), A_{\lambda}(x)\right]\right] } \\
& \times \eta\left(x-x^{\prime}\right) \eta\left(x^{\prime}-x^{\prime \prime}\right),
\end{aligned}
$$

where $H(x)=-j_{\mu}(x) A_{\mu}(x)$ and the $A$ 's are now interaction representation operators. Substituting for $H(x)$,

${ }^{13}$ W. Furry, Phys. Rev. 81, 115 (1951).

14 J. Schwinger, Phys. Rev. 76, 790 (1949). 
and making use of such well known results as

$$
\begin{aligned}
{\left[A_{\lambda}\left(x^{\prime}\right), A_{\sigma}(x)\right] } & =i \delta_{\lambda \sigma} D\left(x-x^{\prime}\right), \\
D_{r}(x) & =-\eta(x) D(x),
\end{aligned}
$$

we find

$$
\begin{aligned}
\mathbf{A}_{\lambda}(x)=A_{\lambda}(x) & +\int_{-\infty}^{\infty} d^{4} x^{\prime} D_{r}\left(x-x^{\prime}\right) j_{\lambda}\left(x^{\prime}\right) \\
& -i \int_{-\infty}^{\infty} d^{4} x^{\prime} \int_{-\infty}^{\infty} d^{4} x^{\prime \prime} A_{\mu}\left(x^{\prime \prime}\right) \\
& \times\left[j_{\mu}\left(x^{\prime \prime}\right), j_{\lambda}\left(x^{\prime}\right)\right] D_{r}\left(x-x^{\prime}\right) \eta\left(x^{\prime}-x^{\prime \prime}\right) .
\end{aligned}
$$

We assume that the usual adiabatic switching on and off procedures are carried out in order to give meaning to the above integrals.

The commutator in Eq. (3.1) may now be evaluated easily, with the result

$$
\begin{aligned}
g_{\lambda \sigma}(x, y)=\delta_{\lambda \sigma} D_{r}(x-y) \delta_{f i} & \\
& +\eta(x-y)\left\langle 0, f\left|G_{\lambda \sigma}(x, y)\right| i, 0\right\rangle,
\end{aligned}
$$

with $G_{\lambda \sigma}(x, y)$ given by

$$
\begin{aligned}
G_{\lambda \sigma}(x, y)=i \int_{-\infty}^{\infty} d^{4} x^{\prime} \int_{-\infty}^{\infty} d^{4} x^{\prime \prime}\left[j_{\lambda}\left(x^{\prime \prime}\right), j_{\sigma}\left(x^{\prime}\right)\right] \\
\quad \times\left\{D_{r}\left(x-x^{\prime}\right) D_{r}\left(y-x^{\prime \prime}\right)\right. \\
+D_{r}\left(x-x^{\prime}\right) D\left(y-x^{\prime \prime}\right) \eta\left(x^{\prime}-x^{\prime \prime}\right) \\
\left.\quad+D\left(x-x^{\prime}\right) D_{r}\left(y-x^{\prime \prime}\right) \eta\left(x^{\prime \prime}-x^{\prime}\right)\right\}
\end{aligned}
$$

In the limit as $x_{0} \rightarrow+\infty, y_{0} \rightarrow-\infty$, only the middle term survives, in accordance with the adiabatic hypothesis mentioned above. In this limit, $G_{\lambda \sigma}(x, y)$ becomes

$$
\begin{aligned}
& G_{\lambda \sigma}(x, y)=i \int_{-\infty}^{\infty} d^{4} x^{\prime} \int_{-\infty}^{\infty} d^{4} x^{\prime \prime}\left[j_{\lambda}\left(x^{\prime \prime}\right), j_{\sigma}\left(x^{\prime}\right)\right] \\
& \times D\left(x-x^{\prime}\right) D\left(x^{\prime \prime}-y\right) \eta\left(x^{\prime}-x^{\prime \prime}\right),
\end{aligned}
$$

where we have used (3.3) and also $D(x)=-D(-x)$. Substituting the Fourier representation of the $D$ 's, namely,

we have

$$
D(x)=\frac{-i}{(2 \pi)^{3}} \int d^{4} k \epsilon(k) \delta\left(k^{2}\right) e^{i k \cdot x}
$$

$$
\begin{aligned}
\left\langle 0, f\left|G_{\lambda \sigma}(x, y)\right| i, 0\right\rangle & =-\int \frac{d^{4} k}{(2 \pi)^{3}} \int \frac{d^{4} k^{\prime}}{(2 \pi)^{3}} e^{i k^{\prime} \cdot x-i k \cdot y} \\
& \times \epsilon(k) \epsilon\left(k^{\prime}\right) \delta\left(k^{2}\right) \delta\left(k^{\prime 2}\right) \mathscr{N} f^{f i}\left(k^{\prime}, k\right),
\end{aligned}
$$

where

$$
\begin{array}{r}
\mathscr{T}_{\lambda \sigma^{f}}{ }^{i}\left(k^{\prime}, k\right)=i \int d^{4} x^{\prime} \int d^{4} x^{\prime \prime}\left\{\left\langle f\left|j_{\lambda}\left(x^{\prime}\right) j_{\sigma}\left(x^{\prime \prime}\right)\right| i\right\rangle\right. \\
\times e^{-i k^{\prime} \cdot x^{\prime}+i k \cdot x^{\prime \prime}} \eta\left(x^{\prime}-x^{\prime \prime}\right)-\left\langle f\left|j_{\sigma}\left(x^{\prime}\right) j_{\lambda}\left(x^{\prime \prime}\right)\right| i\right\rangle \\
\left.\times e^{i k \cdot x^{\prime}-i k^{\prime} \cdot x^{\prime \prime}} \eta\left(x^{\prime \prime}-x^{\prime}\right)\right\}
\end{array}
$$

We write now :

$$
\begin{aligned}
\left\langle b\left|j_{\mu}(x)\right| a\right\rangle= & \left\langle b\left|j_{\mu}(\mathbf{x})\right| a\right\rangle e^{-i\left(E_{a}-E_{b}\right) x_{0}}, \\
\left\langle f\left|j_{\lambda}\left(x^{\prime}\right) j_{\sigma}\left(x^{\prime \prime}\right)\right| i\right\rangle= & \sum_{n}\left\langle f\left|j_{\lambda}\left(\mathbf{x}^{\prime}\right)\right| n\right\rangle\left\langle n\left|j_{\sigma}\left(\mathbf{x}^{\prime \prime}\right)\right| i\right\rangle \\
& \times e^{i E\left(x_{0}^{\prime}-x_{0}^{\prime \prime \prime}\right)} e^{-i E_{n}\left(x_{0}^{\prime}-x_{0}{ }^{\prime \prime}\right)}, \\
\eta(x)= & \frac{1}{2 \pi i} \int_{-\infty}^{\infty} d a \frac{e^{i a x_{0}}}{a-i \epsilon}
\end{aligned}
$$

where $n$ labels a complete set of state vectors for the matter system with the positive energies $E_{n}$ and we have used $E_{f}=E_{i}=E$. The time integrations in (3.10) may be carried out immediately, with the result

$$
\begin{aligned}
& \mathscr{T}_{\lambda_{\sigma}{ }^{f i}\left(k^{\prime}, k\right)=2 \pi \delta\left(k_{0}-k_{0}\right)} \sum_{m} \int d^{3} x^{\prime} \int d^{3} x^{\prime \prime} \\
& \times\left[\frac{\left\langle f\left|j_{\lambda}\left(\mathbf{x}^{\prime}\right)\right| n\right\rangle\left\langle n\left|j_{\sigma}\left(\mathbf{x}^{\prime \prime}\right)\right| i\right\rangle}{E+k_{0}-E_{n}+i \epsilon} e^{-i k^{\prime} \cdot x+i k \cdot x^{\prime \prime}}\right. \\
& \left.+\frac{\left\langle f\left|j_{\sigma}\left(\mathbf{x}^{\prime}\right)\right| n\right\rangle\left\langle n\left|j_{\lambda}\left(\mathbf{x}^{\prime \prime}\right)\right| i\right\rangle}{E-k_{0}-E_{n}-i \epsilon} e^{i k \cdot x^{\prime}-i k \cdot x^{\prime \prime}}\right]
\end{aligned}
$$

This result is to be compared to the matrix element of the $S$ matrix corresponding to a transition in which a photon of four-momentum $k$ and polarization $\sigma$ is scattered into a photon of four-momentum $k^{\prime}$ and polarization $\lambda$ while the matter system makes a transition from the state $i$ to state $f$ of equal energy. Writing $S=1-i R$, we have

$$
\begin{aligned}
& \mathscr{F}_{\lambda \sigma}{ }^{f i}\left(k^{\prime}, k\right)=\left\langle\lambda k^{\prime} f|R| i k \sigma\right\rangle \\
&=--i \int_{-\infty}^{\infty} d^{4} x^{\prime} \int_{-\infty}^{\infty} d^{4} x^{\prime \prime}\left\langle\lambda k^{\prime} f\right| H\left(x^{\prime}\right) \\
& \times H\left(x^{\prime \prime}\right)|i k \sigma\rangle_{\eta}\left(x^{\prime}-x^{\prime \prime}\right) \\
&=-i \int_{-\infty}^{\infty} d^{4} x^{\prime} \int_{-\infty}^{\infty} d^{4} x^{\prime \prime}\left\{\left\langle f\left|j_{\lambda}\left(x^{\prime}\right) j_{\sigma}\left(x^{\prime \prime}\right)\right| i\right\rangle\right. \\
& \quad \times e^{-i k^{\prime} \cdot x^{\prime}+i k \cdot x^{\prime \prime}} \eta\left(x^{\prime}-x^{\prime \prime}\right) \\
&+\left\langle f\left|j_{\sigma}\left(x^{\prime}\right) j_{\lambda}\left(x^{\prime \prime}\right)\right| i\right\rangle \\
& \quad \times e^{\left.-i k^{\prime} \cdot x^{\prime \prime}+i k \cdot x^{\prime} \eta\left(x^{\prime}-x^{\prime \prime}\right)\right\} .}
\end{aligned}
$$

Using Eq. (3.11) as before, we find

$$
\begin{aligned}
\mathscr{F}_{\lambda \sigma}{ }^{f i}\left(k^{\prime}, k\right)=2 \pi \delta\left(k_{0}-k_{0}{ }^{\prime}\right) \sum_{n} \int d^{3} x^{\prime} \int d^{3} x^{\prime \prime} \\
+\left[\frac{\left\langle f\left|j_{\lambda}\left(\mathbf{x}^{\prime}\right)\right| n\right\rangle\left\langle n\left|j_{\sigma}\left(\mathbf{x}^{\prime \prime}\right)\right| i\right\rangle}{E+k_{0}-E_{n}+i \epsilon} e^{-i k^{\prime} \cdot x^{\prime}+i k \cdot x^{\prime \prime}}\right. \\
\left.+\frac{\left\langle f\left|j_{\sigma}\left(\mathbf{x}^{\prime}\right)\right| n\right\rangle\left\langle n\left|j_{\lambda}\left(\mathbf{x}^{\prime \prime}\right)\right| i\right\rangle}{E-k_{0}-E_{n}+i \epsilon} e^{i k \cdot x^{\prime}-i k^{\prime} \cdot x^{\prime \prime}}\right] .
\end{aligned}
$$


We see that except for the sign of $\epsilon$ in the second term, (3.14) and (3.12) are identical. If, for example, $E$ is the ground state of the system, $E_{n}>E$ for all $n$, and $k_{0}>0$ (which is the case for real photon absorption as we have defined it), the difference between the two expressions is of no consequence, because in such a case the second denominator can never vanish. Of course, $k_{0}$ in Eq. (3.12) is not restricted to positive values and we may formally consider $k_{0}$ in Eq. (3.14) taking on negative values. We write for the coefficient of $2 \pi \delta\left(k_{0}-k_{0}{ }^{\prime}\right)$ in the two cases $M_{\lambda \sigma}{ }^{f i}\left(k^{\prime}, k\right)$ and $F_{\lambda \sigma}{ }^{f i}\left(k^{\prime}, k\right)$ for (3.12) and (3.14), respectively, and further introduce the dispersive and absorptive parts of $F_{\lambda \sigma}{ }^{f i}$ according to the definition:

$$
F_{\lambda \sigma}^{f i}\left(k^{\prime} \cdot k\right)=D_{\lambda \sigma}^{f i}\left(k^{\prime}, k\right)+i A_{\lambda \sigma}{ }^{f i}\left(k^{\prime}, k\right),
$$

where the division is made according to whether the intermediate states in the sum are virtual (contributing to $D$ ) or real (contributing to $A$ ). Unless $i=f, \sigma=\lambda$, $D$ and $A$ will generally themselves be complex. We shall see examples of this in Sec. V. In terms of $D$ and $A$, $M$ can be written as

$$
M_{\lambda \sigma}^{f i}\left(k^{\prime}, k\right)=D_{\lambda \sigma}^{f i}\left(k^{\prime}, k\right)+i \epsilon\left(k_{0}\right) A_{\lambda \sigma}^{f i}\left(k^{\prime}, k\right) .
$$

Thus, the factor $\epsilon\left(k_{0}\right)$ in the absorptive term reflects the previously noted differences in the energy denominators. It should be remarked that $F_{\lambda \sigma} f i\left(k^{\prime}, k\right)$ is the scattering amplitude except for some easily supplied factors. For example, in Compton scattering, one must supply a factor of $(1 / 4 \pi)\left(\nu^{\prime} / \nu\right)$ if $F$ is computed in the laboratory system, or a factor of $(1 / 4 \pi)(m / W)$, where $W$ is the total energy if $F$ is computed in the barycentric system.

We now return to Eq. (3.9) which may be written, in virtue of the $\delta$ functions in the integrand and the definition of $M$, as

$$
\begin{aligned}
& \left\langle 0, f\left|G_{\lambda \sigma}(x, y)\right| i, 0\right\rangle \\
& =\int_{-\infty}^{\infty} \frac{d k_{0} k_{0}^{2}}{(2 \pi)^{4}} e^{-i k_{0}\left(x_{0}-y_{0}\right)} \int d \Omega_{\mathbf{n}} \int d \Omega_{\mathbf{n}^{\prime}} \\
& \quad \times e^{i\left|k_{0}\right| \mathbf{n}^{\prime} \cdot \mathbf{x}-i\left|k_{0}\right| \mathbf{n} \cdot \mathrm{y}} M_{\lambda \sigma} i f\left(k_{0} ;\left|k_{0}\right| \mathbf{n}^{\prime} ;\left|k_{0}\right| \mathbf{n}\right) .
\end{aligned}
$$

We have introduced a more explicit notation for the arguments of $M_{\lambda \sigma}$ if. The $k_{0}$ refers to the "photon frequency" that appears in the energy denominators of Eq. (3.12); $\left|k_{0}\right| \mathbf{n}^{\prime}$ was, before the above-mentioned $\delta$ functions were used, the momentum vector $\mathbf{k}^{\prime}$ of the "emitted photon," and similarly $\left|k_{0}\right| \mathbf{n}$ was the momentum vector of the "absorbed photon," k.

We have already imagined that $x_{0} \rightarrow+\infty, y_{0} \rightarrow-\infty$; we now consider removing $\mathbf{x}, \mathbf{y}$ to infinity. As in Sec. II, it is convenient to put $\mathbf{y}=-\mathbf{x}$ and for the purpose of carrying out the integrations over $\mathbf{n}$ and $\mathbf{n}^{\prime}$ we choose the direction $x$ as polar axis, and define a unit vector $\mathbf{n}_{x}$ parallel to $x$. In carrying out the angular integrations, we integrate by parts and retain only the leading terms in powers of $1 /|x|$. We find

$$
\begin{aligned}
& \left\langle 0, f\left|G_{\lambda \sigma}(x, y)\right| i, 0\right\rangle \\
& =-\frac{1}{(2 \pi)^{2}|\mathbf{x}|^{2}} \int_{-\infty}^{\infty} d k_{0} e^{-i k_{0}\left(x_{0}-y_{0}\right)}\left\{e^{2 i k_{0}|\mathbf{x}|}\right. \\
& \quad \times M_{\lambda \sigma}{ }^{i f}\left(k_{0} ; k_{0} \mathbf{n}_{x} ; k_{0} \mathbf{n}_{x}\right)-M_{\lambda \sigma}^{f i}\left(k_{0} ;-\mathbf{n}_{x} k_{0} ; \mathbf{n}_{x} k_{0}\right) \\
& \quad \times M_{\lambda \sigma}^{f i}\left(k_{0} ; \mathbf{n}_{x} k_{0} ;-\mathbf{n}_{x} k_{0}\right)+e^{-2 i k_{0}|\mathbf{x}|} \\
& \left.\quad \times M_{\lambda \sigma}^{f i}\left(k_{0} ;-\mathbf{n}_{x} k_{0} ;-\mathbf{n}_{x} k_{0}\right)\right\}
\end{aligned}
$$

We now proceed exactly as we did in Sec. II: We introduce $z=2|x|-\left(x_{0}-y_{0}\right)$, multiply successively by the exponential factor $e^{-i q_{1} z} e^{-i q_{2} z}$ in each case integrating from $z$ to $\infty$, and use the argument following Eq. (2.24). Imposing our condition that $\langle 0, f|G(x, y)| i \cdot 0\rangle$ vanish for $z>0$ yields, as our final condition,

$$
\int_{-\infty}^{\infty} d k_{0} e^{i k_{0} z} \frac{M_{\lambda \sigma}^{f i}\left(k_{0} ; \mathbf{n} k_{0} ; \mathbf{n} k_{0}\right)}{\left(k_{0}-q_{1}\right)\left(k_{0}-q_{2}\right)}=0, \quad z>0,
$$

where we have dropped the subscript $x$ of $\mathbf{n}_{x}$. This will furnish more general forms of the dispersion relations than are usually considered, since we need not confine ourselves to the completely coherent case $\lambda=\sigma, f=i$. Note that for $k_{0}>0, M_{\lambda \sigma}{ }^{f i}\left(k_{0} ; \mathbf{n} k_{0} ; \mathbf{n} k_{0}\right)$ is, except for a factor $1 / 4 \pi$, the forward scattering amplitude in the laboratory system. (See remarks following (3.16).)

It has not been necessary for us to make any assumptions about the evenness or oddness of $M$ as a function of frequency $k_{0}$ up to this time. The precise dispersion relations which follow from Eq. (3.19) depend, however, on this question. We may deduce the behavior of $M$ directly from its explicit form given in Eq. (3.12). Writing $\mathbf{k}=k_{0} \mathbf{n}, \mathbf{k}^{\prime}=k_{0} \mathbf{n}^{\prime}$ in accordance with Eq. (3.19), we see by inspection of Eq. (3.12) that

$M_{\lambda \sigma^{f i}}\left(-k_{0} ;-k_{0} \mathbf{n}^{\prime} ;-k_{0} \mathbf{n}\right)=M_{\lambda \sigma^{i f}}{ }^{*}\left(+k_{0} ; k_{0} \mathbf{n}^{\prime}, k_{0} \mathbf{n}\right)$.

If we regard $M$ as an operator on the initial and final states, we see that changing $k_{0}$ into minus $k_{0}$ yields the Hermitian conjugate operator. There are similar relations which will not be used here but which are perhaps worth while pointing out. One of these is the direct counterpart of a relation recently used by Gell-Mann and Goldberger ${ }^{15}$ in meson-nucleon scattering, namely

$$
F_{\lambda \sigma^{f}} i\left(k^{\prime}, k\right)=F_{\sigma \lambda}{ }^{f i}\left(-k,-k^{\prime}\right) .
$$

Note that this relation is not true for $M_{\lambda \sigma}{ }^{f i}$. Now the four-vectors $k^{\prime}, k$ in (3.21) refer to real photon fourmomenta, and in the case being considered here $k_{0}^{\prime}=k_{0}$; we may write then $\mathbf{k}=\left|k_{0}\right| \mathbf{n}$ and $\mathbf{k}^{\prime}=\left|k_{0}\right| \mathbf{n}^{\prime}$, since the amplitude $F$ is defined only for positive $k_{0}$ and we are free to decide whether $\mathbf{k}, \mathbf{k}^{\prime}$ shall change sign for

${ }^{15}$ M. Gell-Mann and M. L. Goldberger (to be published). 
$k_{0} \rightarrow-k_{0}$. Writing Eq. (3.21) very explicitly, we have

$$
\begin{aligned}
F_{\lambda \sigma^{f i}}\left(k_{0} ;\left|k_{0}\right| \mathbf{n}^{\prime} ;\left|k_{0}\right| \mathbf{n}\right) \\
\quad=F_{\sigma \lambda}{ }^{f i}\left(-k_{0} ;-\left|k_{0}\right| \mathbf{n} ;-\left|k_{0}\right| \mathbf{n}^{\prime}\right) .
\end{aligned}
$$

It is easy to prove from our explicit formula, Eq. (3.14), that if $\lambda, \sigma=1,2$, or 3 , which is the case for real photons,

$$
\begin{aligned}
& F_{\sigma \lambda}{ }^{f i}\left(-k_{0} ;-\left|k_{0}\right| \mathbf{n} ;-\left|k_{0}\right| \mathbf{n}^{\prime}\right) \\
&= \pm F_{\sigma \lambda}{ }^{f i}\left(-k_{0} ;\left|k_{0}\right| \mathbf{n} ;\left|k_{0}\right| n^{\prime}\right),
\end{aligned}
$$

where one takes the plus or minus sign depending on whether the parities of the initial and final states are the same or different. Thus Eq. (3.22) becomes

$$
\begin{aligned}
& F_{\lambda \sigma}{ }^{f i}\left(k_{0} ;\left|k_{0}\right| \mathbf{n} ;\left|k_{0}\right| \mathbf{n}^{\prime}\right) \\
& = \pm F_{\sigma \lambda} f i\left(-k_{0} ;\left|k_{0}\right| \mathbf{n} ;\left|k_{0}\right| \mathbf{n}^{\prime}\right) .
\end{aligned}
$$

In exactly the same way one proves

$$
\begin{aligned}
& M_{\lambda \sigma^{f i}}\left(-k_{0} ;\left|k_{0}\right| \mathbf{n}^{\prime} ;\left|k_{0}\right| \mathbf{n}\right) \\
& \quad= \pm M_{\lambda \sigma}{ }^{i f^{*}}\left(k_{0} ;\left|k_{0}\right| \mathbf{n}^{\prime} ;\left|k_{0}\right| \mathbf{n}\right) .
\end{aligned}
$$

Our principal result, Eq. (3.19), will be applied in Sec. $V$ to a number of examples. In these applications we never go beyond the $e^{2}$ approximation under which (3.19) was derived.

\section{ANALYTICITY AND DISPERSION RELATIONS}

In the previous sections we have shown that the requirements of commutativity of field operators taken at two space-like points have resulted in restrictions on what are essentially forward-scattering amplitudes of the variety that the functions are the Fourier transforms of other functions which vanish for positive values of their arguments. [See Eqs. (2.26) and (3.19).] The relation between the requirement that a function $h(w)$ be the Fourier transform of a function $g(z)$ which vanishes for positive values of $z$, and dispersion relations of the variety given by Kramers and Kronig, has been discussed in great detail by Toll and van Kampen. We shall give a short description of the mathematical theory, taking advantage of certain simplifications which we may introduce on the basis of physics.

We shall assume that the total cross section for any physical process either approaches a constant for high frequencies or approaches zero. Actually the theory we shall develop will remain valid even if the cross section were to increase with energy, provided this increase were not so rapid that the integral $\int_{0}^{\infty} d \nu \sigma^{2}(\nu) / \nu^{2}$ ceased to exist. With this limitation on the class of functions to be considered, we may use the rather weaker theorems from Fourier integral theory relating to functions which are square integrable.

In terms of the scattering amplitude $f(\nu)$, our assumption on the high-frequency dependence of the cross sections becomes

$$
\int^{\infty} d \nu\left(\frac{\operatorname{Im} f(\nu)}{\nu^{2}}\right)^{2}<\infty
$$

[We used the well-known relation $\sigma(\nu)=4 \pi \operatorname{Im} f(\nu) / \nu$.] We shall assume that $\operatorname{Re} f(\nu)$ satisfies the same requirement. In order to avoid difficulties at $\nu=0$ we shall consider the conditions which may be imposed on a function $h(v)$ [which in our application is essentially the forward-scattering amplitude, and which we now assume has the boundedness property expressed in Eq. (4.1)] from the requirement

$$
g(z) \equiv \int_{-\infty}^{\infty} d \nu \frac{h(\nu)}{\left(\nu-q_{1}\right)\left(\nu-q_{2}\right)} e^{i \nu z}=0, \quad z>0 .
$$

The frequencies $q_{1}$ and $q_{2}$ are complex with negative imaginary parts which will later be allowed to approach zero, and whose real parts will be chosen conveniently. The integrand in Eq. (4.2), $h(\nu) /\left(\nu-q_{1}\right)\left(\nu-q_{2}\right)$ may be expressed in terms of $g(z)$ by the Fourier theorem according to

$$
\frac{h(\nu)}{\left(\nu-q_{1}\right)\left(\nu-q_{2}\right)} \equiv \bar{h}(\nu)=\frac{1}{2 \pi} \int_{-\infty}^{0} d z g(z) e^{-i \nu z} .
$$

From our original restrictions $h(\nu)$ we know that this integral is convergent for all values of $\nu$. If we add to $\nu$ a positive imaginary part $i \gamma$ the integrand is multiplied by $e^{\gamma z}$ and the integral consequently converges even better. It follows that $\bar{h}(\nu)$ is the boundary value of a function which is analytic in the entire upper half $\nu$ plane. We now remark that not only is $\bar{h}(\nu)$ analytic, but it is also square integrable in the upper half-plane. To prove this, we observe that

$$
\begin{aligned}
& \int_{-\infty}^{\infty} d \nu|\bar{h}(\nu+i \gamma)|^{2}=\frac{1}{2 \pi} \int_{-\infty}^{0} d z e^{2 \gamma z}|g(z)|^{2} \\
& \leqslant \int_{-\infty}^{\infty} d \nu|\bar{h}(\nu)|^{2}<\infty,
\end{aligned}
$$

independent of $\gamma$. Evidently the analyticity of $\bar{h}(\nu)$ implies that of $h(v)$, but of course not the integrability.

We wish now to express our requirement (4.2) as a dispersion relation of the familiar variety. This may be done in a variety of ways; one simple way is as follows: Let us simply evaluate the integral

$$
\frac{\mathrm{P}}{i \pi} \int_{-\infty}^{\infty} d \nu^{\prime} \frac{\bar{h}\left(\nu^{\prime}\right)}{\nu^{\prime}-\nu}
$$

where $\mathrm{P}$ stands for the Cauchy principle value. Since $\bar{h}(\nu)$ has no poles in the upper half-plane, and because of (4.4), ${ }^{16}$ the integral in (4.5) may be evaluated imme-

${ }^{16}$ It can be shown that Eq. (4.4) implies that the integral over an infinite semicircle above the real axis vanishes. 
diately by contour integration, and it yields

$$
\bar{h}(\nu)=\frac{\mathrm{P}}{i \pi} \int_{-\infty}^{\infty} d \nu^{\prime} \frac{\bar{h}\left(\nu^{\prime}\right)}{\nu^{\prime}-\nu}
$$

Let us now put in our explicit form for $\bar{h}(\nu)$ [Eq. (4.3)], taking $q_{1}=\nu_{1}-i \epsilon_{1}, q_{2}=i \epsilon_{2}$, and considering the limit as $\epsilon_{1}$ and $\epsilon_{2}$ approach zero. [Recall that $1 /(x+i \epsilon)=\mathrm{P}(1 / x)$ $-i \pi \delta(x)$.$] We find that$

$$
\begin{aligned}
& \frac{h(\nu)}{\nu\left(\nu-\nu_{1}\right)}-\frac{1}{\nu_{1}}\left[\frac{h\left(\nu_{1}\right)}{\nu-\nu_{1}}-\frac{h(0)}{\nu}\right] \\
&=\frac{\mathrm{P}}{i \pi} \int_{-\infty}^{\infty} d \nu^{\prime} \frac{h\left(\nu^{\prime}\right)}{\nu^{\prime}\left(\nu^{\prime}-\nu_{1}\right)\left(\nu^{\prime}-\nu\right)}
\end{aligned}
$$

where the principal value sign applies to all three singularities of the integrand. In our applications it will be convenient to consider the limit of both sides as $\nu_{1} \rightarrow 0$. Under these circumstances, we find

$\frac{h(\nu)}{\nu^{2}}-\frac{h^{\prime}(0)}{\nu}-\frac{h(0)}{\nu^{2}}=\lim _{\nu_{1} \rightarrow 0} \frac{\mathrm{P}}{i \pi} \int_{-\infty}^{\infty} d \nu^{\prime} \frac{h\left(\nu^{\prime}\right)}{\nu^{\prime}\left(\nu^{\prime}-\nu_{1}\right)\left(\nu^{\prime}-\nu\right)}$.

There are two special cases of interest: (1) $h(\nu)$ $=h^{*}(-\nu)$, and (2) $h(\nu)=-h^{*}(-\nu)$. In the first case, we have

$$
\begin{aligned}
& \operatorname{Re} h(\nu)=\frac{2 \nu^{2}}{\pi} \int_{0}^{\infty} d \nu^{\prime} \frac{\operatorname{Im} h\left(\nu^{\prime}\right)}{\nu^{\prime}\left(\nu^{\prime 2}-\nu^{2}\right)} \\
& +\lim _{\nu 1 \rightarrow 0} \frac{2 \nu^{2} \nu_{1}\left(\nu_{1}+\nu\right)}{\pi} \mathbf{P} \int_{0}^{\infty} d \nu^{\prime} \frac{\operatorname{Im} h\left(\nu^{\prime}\right)}{\nu^{\prime}\left(\nu^{\prime 2}-\nu_{1}^{2}\right)\left(\nu^{\prime 2}-\nu_{1}\right)}
\end{aligned}
$$

whereas, in the second case,

$\operatorname{Re} h(\nu)-\nu h^{\prime}(0)=\frac{2 \nu^{2}}{\pi} \lim _{\nu_{1} \rightarrow 0} \int_{0}^{\infty} d \nu^{\prime} \frac{\left(\nu+\nu_{1}\right) \operatorname{Im} h\left(\nu^{\prime}\right)}{\left(\nu^{\prime 2}-\nu_{1}^{2}\right)\left(\nu^{\prime 2}-\nu^{2}\right)}$.

In all of our applications we may pass to the limit of $\nu_{1} \rightarrow 0$ without difficulty because $\operatorname{Im} h(\nu)$ will invariably vanish for $\nu$ less than some threshold value. Equations (4.9) and (4.10) become then, respectively,

$$
\begin{aligned}
\operatorname{Re} h(\nu)-h(0) & =\frac{2 \nu^{2}}{\pi} \mathrm{P} \int_{0}^{\infty} d \nu^{\prime} \frac{\operatorname{Im} h\left(\nu^{\prime}\right)}{\nu^{\prime}\left(\nu^{\prime 2}-\nu^{2}\right)} \\
\operatorname{Re} h(\nu)-\nu h^{\prime}(0) & =\frac{2 \nu^{3}}{\pi} \mathrm{P} \int_{0}^{\infty} d \nu^{\prime} \frac{\operatorname{Im} h\left(\nu^{\prime}\right)}{\nu^{\prime 2}\left(\nu^{\prime 2}-\nu^{2}\right)} .
\end{aligned}
$$

Case (1) in (4.11) becomes exactly the Kramers-Kronig dispersion relation provided we identify $h(\nu)$ with the quantity $N_{\lambda \sigma}$ if of Sec. III and consider the case of complete coherence, which we notice satisfies the appropriate condition $h(\nu)=h^{*}(\nu)$ [compare Eq. (3.20)]. Using the previously mentioned relation between the imaginary part of the coherent scattering amplitude and the total cross section, we have the result [in the notation of Eq. (3.15)]:

$$
\begin{aligned}
D_{\lambda \lambda} i i(\nu)-D_{\lambda \lambda} i i(0) & =\frac{2 \nu^{2}}{\pi} \mathrm{P} \int_{0}^{\infty} d \nu^{\prime} \frac{A_{\lambda \lambda} i i\left(\nu^{\prime}\right)}{\nu^{\prime}\left(\nu^{\prime 2}-\nu^{2}\right)} \\
& =\frac{\nu^{2}}{-\pi^{2}} \mathrm{P} \int_{0}^{\infty} d \nu^{\prime} \frac{\sigma\left(\nu^{\prime}\right)}{\nu^{\prime 2}-\nu^{2}}
\end{aligned}
$$

It should be remembered that our derivation was valid only to order $e^{2}$.

Before passing on to the applications of greatest interest, there are several simple observations worth making. Suppose we apply Eq. (4.12) to the case of a free electron. There is no $e^{2}$ cross section in such a case, and we see that coherent dispersive forward scattering amplitude is independent of frequency and takes on the value $D_{\lambda \lambda} i i(0)=-e^{2} / m$ which is, of course, correct. Consider now the limit of (4.12) as the frequency approaches infinity:

$$
D_{\lambda \lambda}{ }^{i i}(\infty)-D_{\lambda \lambda} i i(0)=-\frac{1}{2 \pi^{2}} \int_{0}^{\infty} d \nu^{\prime} \sigma\left(\nu^{\prime}\right) .
$$

Either both sides are finite or they are both infinite. To order $e^{2}$, for a free electron, both sides are zero; the relation also checks to order $e^{4}$, incidentally. If the scattering is from a bound electron, say in a hydrogen atom, $D_{\lambda \lambda}{ }^{i i}(0)=0$, since one must have Rayleigh scattering at low frequencies. ${ }^{17}$ One might expect $D_{\lambda \lambda}{ }^{i i}(\infty)$ to equal its value for a free electron, $-e^{2} / m$, which would yield the remarkable result:

$$
\int_{0}^{\infty} d \nu \sigma(\nu)=2 \pi^{2} e^{2} / m
$$

which is the well known Thomas-Reiche-Kuhn summation formula usually derived under the assumption of dipole transitions only and $\nu R \ll 1$. Unfortunately, there is some uncertainty as to whether $D_{\lambda \lambda}{ }^{i i}(\infty)$ is equal to the value for a free electron. As has been pointed out by Toll ${ }^{6}$ in the same connection, the photoelectric cross section for large frequencies goes like $1 / \nu$ and this gives rise to a logarithmic frequency dependence in the dispersive amplitude. It is not completely clear that the published calculations of the photoeffect are reliable at very high frequencies. None of the existing direct calculations of the forward scattering amplitude are sufficiently accurate to decide if $D_{\lambda \lambda}{ }^{i i}(\infty)$ takes the free electron value and this matter is being looked into further.

The causality condition (4.2) is not fulfilled for the scattering of light by a classical electron, as has been noted by Toll. ${ }^{6}$ The same criticism applies to the scat-

\footnotetext{
17 Note that the coefficient of the $\nu^{2}$ Rayleigh scattering amplitude is simply $\left(1 / 2 \pi^{2}\right) \int d \nu^{\prime} \sigma\left(\nu^{\prime}\right) / \nu^{\prime 2}$, where $\sigma$ is the atomic absorption cross section for light. This is, of course, an old result, contained in the Kramers-Heisenberg dispersion formula.
} 
tering formula obtained by Heitler's radiation damping theory. It turns out that in those cases the scattering amplitude has a pole in the upper half-plane. It gives a nonvanishing contribution to the commutator outside the light-cone which decays exponentially with a halfwidth of the order of the classical electron radius. This seems to be connected with the well known preacceleration of the classical electron.

It is important to note that one cannot in general expect to have a relation such as

$$
\operatorname{Re} f(\nu, \vartheta)-f(0, \vartheta)=\frac{2 \nu^{2}}{\pi} \mathrm{P} \int_{0}^{\infty} \frac{\operatorname{Im} f\left(\nu^{\prime}, \vartheta\right)}{\nu^{\prime}\left(\nu^{\prime 2}-\nu^{2}\right)}
$$

where $f(\nu, \vartheta)$ is the entire scattering amplitude valid for all angles $\vartheta$. We consider only the case of zero mass. For if this were true, one would have such a relation for the individual $f_{l}$, which in turn leads to the conclusion that the phase shifts $\delta_{l}$ have for small frequencies a frequency dependence of the form $\delta_{0} \sim \nu, \delta_{l} \sim \nu^{3}$ for $l>0$. Such a situation leads to grave difficulties in that the integral in Eq. (2.22) is badly divergent near $\nu \sim 0$. This is because the higher terms in the expansion in powers of $1 / R$ carry also powers of $1 / \nu$ at the same time. The weakest frequency dependence which can be tolerated is $\delta_{l} \sim \nu^{2 l}$. (One customarily has $\delta_{l} \sim \nu^{2 l+1}$ for scattering by a potential; it has been shown by van Kampen that in the limit of a point scatterer $\delta_{l} \sim \nu$.) The situation seems to be that although $f(\nu, \vartheta)$ is analytic in the upper half-plane, it does not have the requisite boundedness for a relation such as (4.15) to obtain. The proof of the above statements about the frequency dependence of the phase shifts is as follows: Assume that

$$
\operatorname{Re} f_{l}(\nu)=\frac{2 \nu^{2}}{\pi} \mathrm{P} \int_{0}^{\infty} d \nu^{\prime} \frac{\operatorname{Im} f_{l}\left(\nu^{\prime}\right)}{\nu^{\prime}\left(\nu^{\prime 2}-\nu^{2}\right)}
$$

and that $\sin ^{2} \delta_{l} / \nu^{2} \rightarrow 0$ for $\rightarrow 0$ at least as rapidly as $\nu^{2}$. We may write (4.16) as

$$
\frac{\sin \delta_{l}(\nu) \cos \delta_{l}(\nu)}{\nu}=\frac{2 \nu^{2}}{\pi} \mathrm{P} \int_{0}^{\infty} d \nu^{\prime} \frac{\sin ^{2} \delta_{l}\left(\nu^{\prime}\right) / \nu^{\prime 2}}{\nu^{\prime 2}-\nu^{2}} .
$$

We now remark that

$$
\mathrm{P} \int_{0}^{\infty} d \nu^{\prime} \frac{1}{\nu^{\prime 2}-\nu^{2}}=\frac{\pi^{2}}{4} \delta(\nu)
$$

so that we may write, setting $\sin ^{2} \delta_{l} / \nu^{2}=s^{2}(\nu), \cos \delta_{l}(\nu)$ $=c(\nu)$,

$$
s(\nu) c(\nu)=\frac{2 \nu^{2}}{\pi} \int_{0}^{\infty} d \nu^{\prime} \frac{s^{2}\left(\nu^{\prime}\right)-s^{2}(\nu)}{\nu^{\prime 2}-\nu^{2}} .
$$

We no longer need the principle value sign since the singularity has been removed. We wish now to show that the integral in (4.19) has a nonzero limit as $\nu \rightarrow 0$ and consequently that the low-frequency behavior of $s(\nu) c(\nu)$ is given by the explicit factor of $\nu^{2}$. We write

$$
\begin{array}{rl}
\int_{0}^{\infty} d \nu^{\prime} \frac{s^{2}\left(\nu^{\prime}\right)-s^{2}(\nu)}{\nu^{\prime 2}-\nu^{2}}=\int_{0}^{\nu_{0}} & d \nu^{\prime} \frac{s^{2}\left(\nu^{\prime}\right)-s^{2}(\nu)}{\nu^{\prime 2}-\nu^{2}} \\
& +\int_{\nu_{0}}^{\infty} \frac{d \nu^{\prime} \frac{s^{2}\left(\nu^{\prime}\right)-s^{2}(\nu)}{\nu^{\prime 2}-\nu^{2}}}{}
\end{array}
$$

where $\nu_{0}$ is a small frequency but greater than $\nu$. Since this is the case, $\nu$ can certainly be put equal to zero in the second integral; and in the first integral, an expansion of $s^{2}\left(\nu^{\prime}\right)$ and $s^{2}(\nu)$ into a power series shows that one may safely pass to the limit of $\nu=0$ in it also. We have then, using $s(0)=0$, the result that

$$
\lim _{\nu \rightarrow 0} \mathrm{P} \int_{j}^{\infty} d \nu^{\prime} \frac{s^{2}\left(\nu^{\prime}\right)}{\nu^{\prime 2}-\nu^{2}}=\int_{j}^{\infty} d \nu^{\prime} \frac{s^{2}\left(\nu^{\prime}\right)}{\nu^{\prime 2}}>0 .
$$

\section{APPLICATIONS}

We shall discuss several applications of the dispersion relations to practical situations. We consider first the case of the scattering of photons by a nucleus. The absorptive phenomena to be considered are photonuclear reactions and meson production. Our technique is to compare the dispersion relations for the nucleus to those of a collection of $Z$ free protons and $N$ free neutrons. We call the nuclear absorption cross section $\sigma_{A}$ and the meson production cross sections for free nucleons $\sigma_{N}$ and $\sigma_{P}$ for neutrons and protons, respectively. (Notice that we are working to order $e^{2}$ only and hence need only $e^{2}$ cross sections.) The corresponding dispersive forward amplitudes are called $D_{A}, D_{P}$, and $D_{N}$. We have from Eq. (4.12) (dropping the subscripts and superscripts on the $D$ 's), subtracting from $D_{A}(\nu)$ the quantity $Z D_{P}(\nu)+N D_{N}(\nu)$,

$$
\begin{gathered}
D_{A}(\nu)-D_{A}(0)-Z\left[\left(D_{P}(\nu)-D_{P}(0)\right]-N\left[D_{N}(\nu)-D_{N}(0)\right]\right. \\
=\frac{\nu^{2}}{2 \pi^{2}} \mathrm{P} \int_{0}^{\infty} d \nu^{\prime} \frac{\sigma_{A}\left(\nu^{\prime}\right)-\left[Z \sigma_{P}\left(\nu^{\prime}\right)+N \sigma_{N}\left(\nu^{\prime}\right)\right]}{\nu^{\prime 2}-\nu^{2}}
\end{gathered}
$$

The cross sections $\sigma_{N}$ and $\sigma_{P}$ are of course zero for frequencies less than the meson mass $\mu$ (or with recoil corrections about $155 \mathrm{Mev}$ ). To evaluate the left-hand side of (5.1), we first note that at zero frequency we have pure Thomson scattering, ${ }^{18}$ so that

$$
\begin{gathered}
D_{N}(0)=0, \quad D_{P}(0)=-e^{2} / M, \\
D_{A}(0)=-(Z e)^{2} / A M .
\end{gathered}
$$

We next assume that in the limit of very large frequencies the scattering amplitude for the nucleus approaches that of equal number of free particles:

$$
\lim _{\nu \rightarrow \infty}\left[D_{A}(\nu)-Z D_{P}(\nu)-N D_{N}(\nu)\right]=0 .
$$

${ }^{18}$ W. Thirring, Phil. Mag. 41, 1193 (1950); N. Kroll and M. Ruderman, Phys. Rev. 93, 233 (1954); Dser, Thirring, and Goldberger, Phys. Rev. 94, 711 (1954). 
This conjecture cannot be verified in any simple way. As has already been pointed out, it appears not to be fulfilled for the scattering of photons by bound electrons. Aside from a logarithmic term in that case undoubtedly associated with the Coulomb field, the corrections for binding are said to be of the order of binding energy divided by electron mass..$^{19}$ Even if these binding effects actually exist in the present case, we would expect them to be of the order of binding energy/ $M$ or about a few percent and we shall neglect them here.

Above the threshold for meson production, the absorption cross section for a nucleus is mainly due to meson production. There are, of course, still some photonuclear processes which one would expect to have a rapidly decreasing cross section as the energy increases, and we shall neglect these contributions to the absorption when $\nu>\mu$. The average cross section per nucleon for a photomeson production from a nucleus is reduced in comparison to that of a free nucleon by ${ }^{20}$ (a) the effect of binding, (b) Pauli principle, (c) reabsorption of produced mesons. The effect (c) essentially cancels out here since we ask for the total absorption cross section. In accordance with the above discussion we write for $\nu>\mu$,

$$
\begin{gathered}
{\left[Z \sigma_{P}(\nu)+N \sigma_{N}(\nu)-\sigma_{A}(\nu)\right]} \\
\equiv\left[Z \sigma_{P}(\nu)+N \sigma_{N}(\nu)\right][1-R(\nu)], \\
R(\mu)=0, \quad R(\infty)=1 .
\end{gathered}
$$

The reduction factor $R(\nu)$ defined by the foregoing equations, because of effects (a) and (b) may be determined by existing experiments to about 20 percent. If now in Eq. (5.1) we substitute Eqs. (5.2), (5.3), and (5.4), and consider the limit as $\nu \rightarrow \infty$, we obtain

$$
\begin{aligned}
\frac{1}{2 \pi^{2}} \int_{\nu}^{\mu} d \nu \sigma_{A}(\nu) & =\frac{Z N}{A} \frac{e^{2}}{M}+\frac{1}{2 \pi^{2}} \int_{\mu}^{\infty} d \nu \\
& \times[1-R(\nu)]\left[Z \sigma_{P}(\nu)+N \sigma_{N}(\nu)\right] .
\end{aligned}
$$

The structure of this sum rule may be understood in the following way: If the meson mass were infinite, so that there were no meson production, real or virtual, then Eq. (5.5) would reduce

$$
\frac{1}{2 \pi^{2}} \int_{0}^{\infty} d \nu \sigma_{A 0}(\nu)=\frac{Z N}{A} \frac{e^{2}}{M}
$$

where $\sigma_{A 0}(\nu)$ is the cross section for photodisintegration of the nucleus. This is precisely the sum rule given by Bethe and Levinger for electric dipole transitions only, in the case of ordinary forces between particles. In our derivation, the term $Z N e^{2} / A M$ in Eq. (5.5) may be regarded as the contribution to the integrals of the

${ }^{19}$ J. S. Levinger, Phys. Rev. 87, 656 (1952).

${ }^{20}$ H. Feshbach and M. Lax, Phys. Rev. 81, 189 (1951); W. Thirring, Helv. Phys. Acta 26, 465 (1953). absorption cross section arising from strictly nonmesonic processes, for in the absence of mesons the infinite-frequency forward-scattering amplitudes for free protons and neutrons would be expected to be $-e^{2} / M$ and zero, respectively. The second term in Eq. (5.5) presumably contains all of the mesonic effects such as modifications of the nucleon currents, exchange forces, etc.

We have evaluated the right-hand side of Eq. (5.5) using the available experimental data and assuming that $\sigma_{N} / \sigma_{P} \sim 1.3$ in the important energy region between $\mu$ and $2 \mu$. We find

$$
\frac{1}{2 \pi^{2}} \int_{0}^{\mu} d \nu \sigma_{A}(\nu)=\frac{Z N}{A} \frac{e^{2}}{M}\left[1+0.1 \frac{A^{2}}{N Z}\right] .
$$

The term $0.1 A^{2} / N Z$ may be assumed accurate to about 30 percent on the basis of the present data. This is to be compared with the Bethe-Levinger sum rule,

$$
\frac{1}{2 \pi^{2}} \int_{0}^{\infty} d \nu \sigma_{D}(\nu)=\frac{Z N}{A} \frac{e^{2}}{M}(1+0.8 x)
$$

where $\sigma_{D}(\nu)$ is the electric dipole absorption cross section and $x$ is the percentage of exchange forces in the two-body forces assumed to act between nucleons. Our sum rule, Eq. (5.7), has been derived under rather more general conditions than that of Bethe and Levinger, Eq. (5.8), and would appear to have some advantages over it: (1) All multiples are included automatically, (2) no assumptions whatsoever are made about nuclear forces or nuclear wave functions, (3) the upper limit of integration is clearly specified in (5.7), whereas $\sigma_{D}$ in Eq. (5.8) can be regarded as the total nuclear absorption cross section only below the meson threshold, and finally, (4) only experimental quantities appear in (5.7) in comparison with the $0.8 x$ in (5.8) which is a very "model-dependent" quantity. It is perhaps worth repeating that our only assumptions were that the forward-scattering amplitude for a bound nucleon approach that of a free nucleon for infinitefrequency photons, and that the absorption cross section for photonuclear processes not involving meson production may be neglected for photon frequencies above the threshold. (Simple estimates of direct nucleon ejection cross sections at these energies indicate that this neglect is well justified.) There are at present no experimental data for making an accurate test of Eq. (5.7). One finds by comparison with experiments with a maximum energy of about $50 \mathrm{Mev}$ that there must be considerable absorption in the 50- to $150-\mathrm{Mev}$ region, and there are practically no data in this energy range. It would be very useful to have the photodisintegration cross section for deuterium up to the threshold since the data on meson production in deuterium is quite extensive. For the time being, Eq. (5.7) can perhaps best be used to check the consistency of experimental 
data, since it is almost surely accurate to within 30 percent.

Our next application is to a study of the scattering of $\gamma$ rays by protons. The amplitude for the forward scattering of photons from linear polarization state $\lambda$ to state $\mu$ by a proton (or generally, any spin $\frac{1}{2}$ particle) must be of the general form

$$
F_{\mu \lambda}(\nu)=f_{1}(\nu) \mathbf{e}_{\mu} \cdot \mathbf{e}_{\lambda}+i f_{2}(\nu) \boldsymbol{\sigma} \cdot \mathbf{e}_{\mu} \times \mathbf{e}_{\lambda},
$$

where $\mathbf{e}_{\mu}, \mathbf{e}_{\lambda}$ are the photon polarization vectors and $\boldsymbol{\sigma}$ is the spin matrix of the proton. (We are regarding $F_{\mu \lambda}$ as a matrix in spin space.) The factor $i$ is included so that if the frequency is below the threshold of particle production, $f_{2}(\nu)$ would be real. In general, of course, both $f_{1}(\nu)$ and $f_{2}(\nu)$ are complex, having dispersive and absorptive parts. From the scattering amplitude $F_{\mu \lambda}(\nu)$ given above, we construct the quantity $M_{\mu \lambda}$ of Sec. III (for forward scattering only) which differs from $F_{\mu \lambda}$ by having the absorptive parts of $f_{1}$ and $f_{2}$ multiplied by $\epsilon(\nu)$. Thus, writing $f_{1}=d_{1}+i a_{1}, f_{2}=d_{2}$ $+i a_{2}$, we have

$$
\begin{aligned}
M_{\mu \lambda}(\nu)=\left[d_{1}(\nu)\right. & \left.+i \epsilon(\nu) a_{1}(\nu)\right] e_{\mu} \cdot e_{\lambda} \\
& +i\left[d_{2}(\nu)+i \epsilon(\nu) a_{2}(\nu)\right] \boldsymbol{\sigma} \cdot \mathbf{e}_{\mu} \times \mathbf{e}_{\lambda} .
\end{aligned}
$$

The behavior of the various functions for negative values of the frequency is deduced from the general requirements, Eq. (3.20),

We deduce that

$$
M_{\mu \lambda}(\nu)=M_{\mu \lambda}^{\dagger}(-\nu) .
$$

$$
\begin{array}{ll}
d_{1}(\nu)=d_{1}(-\nu), & a_{1}(\nu)=a_{1}(-\nu), \\
d_{2}(\nu)=-d_{2}(-\nu), & a_{2}(\nu)=-a_{2}(-\nu) .
\end{array}
$$

Note that in the $e^{2}$ approximation, the $d$ 's and $a$ 's are real.

With this knowledge of the behavior of the functions for negative frequencies, we may apply the considerations of Sec. IV to the entire operator $M_{\mu \lambda}(\nu)$. If we apply our fundamental relation (4.11) to $M_{\mu \lambda}(\nu)$ given by (5.10), we notice that the coefficients of $\mathbf{e}_{\mu} \cdot \mathbf{e}_{\lambda}$ and $i \boldsymbol{\sigma} \cdot \mathbf{e}_{\mu} \times \mathbf{e}_{\lambda}$ separately satisfy the condition as may be seen by taking the trace of the entire expression. Using (5.12) we may write, finally,

$$
\begin{gathered}
d_{1}(\nu)-d_{1}(0)=\frac{2 \nu^{2}}{\pi} \int_{0}^{\infty} d \nu^{\prime} \frac{a_{1}\left(\nu^{\prime}\right)}{\nu^{\prime}\left(\nu^{\prime 2}-\nu^{2}\right)} \\
d_{2}(\nu)-\nu d_{2}^{\prime}(0)=\frac{2 \nu^{3}}{\pi} \int_{0}^{\infty} d \nu^{\prime} \frac{a_{2}\left(\nu^{\prime}\right)}{\nu^{\prime 2}\left(\nu^{\prime 2}-\nu^{2}\right)}
\end{gathered}
$$

[The passage of the limit of $\nu_{1}$ going to zero gives no trouble because both $a_{1}(\nu)$ and $a_{2}(\nu)$ are zero for zero frequency (in our $e^{2}$ approximation) and the zero lower limit in (5.13) is purely formal.]

Now it should be noted that only the quantity $a_{1}(\nu)$ bears any simple relation to the total cross section for $\gamma$ rays, since it is only the coherent amplitude $\mu=\lambda$ that enters in the relation between the imaginary part of the scattering amplitude and the total cross section for unpolarized light. Thus the first of the relations (5.2) may be written (using $d_{1}(0)=-e^{2} / M$ ) as

$$
d_{1}(\nu)=-e^{2} / M+\frac{\nu^{2}}{2 \pi^{2}} \int_{0}^{\infty} d \nu^{\prime} \frac{\sigma\left(\nu^{\prime}\right)}{\nu^{\prime 2}-\nu^{2}}
$$

where $\sigma(\nu)$ is the total cross section to order $e^{2}$ and is thus composed of such processes as electron pair production, meson production, etc. This is the relation used in the first part of this section. The quantity $a_{2}(\nu)$ is not an experimental one in ordinary circumstances, but it can be given an experimental interpretation. If one introduces circular polarization for the photons (see Appendix B) one may define two coherent forward amplitudes corresponding to the cases where the polarization is parallel to the direction of the proton spin $f_{p}$ and antiparallel $f_{a}$. (Note that there is no spin flip in the forward amplitude.) In terms of these coherent amplitudes we may write [in the notation of Eq. (5.9)],

$f_{1}(\nu)=\frac{1}{2}\left[f_{p}(\nu)+f_{a}(\nu)\right], \quad f_{2}(\nu)=\frac{1}{2}\left[f_{p}(\nu)-f_{a}(\nu)\right]$,

and thus, since $f_{p}$ and $f_{a}$ are coherent amplitudes,

$$
\begin{aligned}
& a_{1}(\nu)=\frac{\nu}{4 \pi} \frac{\sigma_{p}(\nu)+\sigma_{a}(\nu)}{2}=\frac{\nu}{4 \pi} \sigma_{\text {unpolarized }}, \\
& a_{2}(\nu)=\frac{\nu}{4 \pi} \frac{\sigma_{p}(\nu)-\sigma_{a}(\nu)}{2}
\end{aligned}
$$

where $\sigma_{p}$ and $\sigma_{a}$ are the total cross sections (or in the $e^{2}$ approximation, the various production cross sections) for photons polarized parallel or antiparallel to the proton spin.

Since the quantities $\sigma_{p}$ and $\sigma_{a}$ are not known experimentally, we cannot predict the forward scattering amplitude for $\gamma$ rays with any real assurance, but can give only a lower limit, based on the neglect of $f_{2}$. In spite of the difficulties associated with making precise predictions, we felt that it would be worth while to pursue the problem further, going, as we shall see shortly, beyond the famework of our causality considerations when necessary. Before turning to this analysis, it should be noted that the relations (5.13) are of some theoretical interest since it is usually far easier to compute theoretically, from any model the absorptive terms $a_{1}$ and $a_{2}$ than to carry out a direct calculation of the dispersive amplitude. This point is well illustrated by the work of Rohrlich and Gluckstern. ${ }^{21}$

In our analysis of the $\gamma$-ray scattering, we shall consider the absorption cross section to be almost ex-

${ }^{21}$ R. Gluckstern and F. Rohrlich, Phys. Rev. 86, 1 (1952). 
clusively the cross section for meson production. One should, of course, include the Delbrück scattering in order to obtain the total coherent scattering amplitude and we shall make some remarks about this later. We have used the data on photomeson production compiled by $\mathrm{Nambu}^{22}$ to compute the dispersive and absorptive parts of the amplitude $f_{1}(\nu)$, according to (5.14) and $a_{1}(\nu)=(\nu / 4 \pi) \sigma(\nu)$. These functions are shown in Fig. 2 . One sees that below the meson threshold, there is destructive interference between the Thomson amplitude and the anomalous amplitude so that the total forward scattering is quite small. The destructive interference arises from the fact that the meson amplitude is negative whereas the anomalous amplitude is positive, being caused by intermediate states with energies greater than the initial state. This is unfortunate since in principle we would like to use this result as a limitation on the phase shifts for $\gamma$-ray scattering below threshold: In the Appendix, a phase shift analysis is presented in terms of which the forward scattering amplitude may be expressed. Assuming that at low energies only a few multipoles need be considered (see below) and that the phase shifts are small, Eq. (5.14) for $d_{1}(\nu)$ gives a limitation on a certain combination of phase shifts. The cancellation just mentioned would not be expected at other angles; for example, if there are only multipoles other than those occurring in Thomson scattering $\left(E_{\frac{3}{2}}{ }^{1}, E_{\frac{1}{2}}{ }^{1}\right)$ there must be constructive interference at other angles, since the total cross section must be larger than the Thomson cross section.

In order to illustrate the general features one might expect experimentally, we have made a calculation of the angular dependence and energy dependence of the scattering of photons by protons on the basis of a model suggested by the phenomenological analysis of photoproduction data. ${ }^{23}$ At this point, of course, we must go far beyond our general considerations and the following discussion should be regarded only as giving qualitative features. The phase shift analysis given in the Appendix has been used with the following values for the phase shifts :

$$
\begin{aligned}
\operatorname{Re} \delta_{\frac{3}{2}} E_{1} & =-\frac{2 \nu}{3} \frac{e^{2}}{M}, \quad \operatorname{Re} \delta_{\frac{1}{2}} E_{1}=-\frac{2 \nu}{3} \frac{e^{2}}{M}\left(1-0.5 \nu^{2}\right), \\
\operatorname{Re} \delta_{\frac{3}{2}} M_{1} & =\frac{2 \nu}{3} \frac{e^{2}}{M} 1 \nu^{2}, \quad \operatorname{Im} \delta_{\frac{3}{2}} E_{1}=0, \\
\operatorname{Im} \delta_{\frac{1}{2}} E_{1}= & 1.4 q \nu \frac{e^{2}}{M}, \quad \nu>1 \quad \operatorname{Im} \delta_{\frac{3}{2}}^{M M_{1}}=1.4 q^{3} \frac{e^{2}}{M}, \quad \nu>1 \\
& =0, \quad \nu<1, \quad=0, \quad \nu<1,
\end{aligned}
$$

${ }^{22}$ Y. Nambu (unpublished).

${ }^{23}$ K. Brueckner and K. Watson, Phys. Rev. 86, 926 (1952).

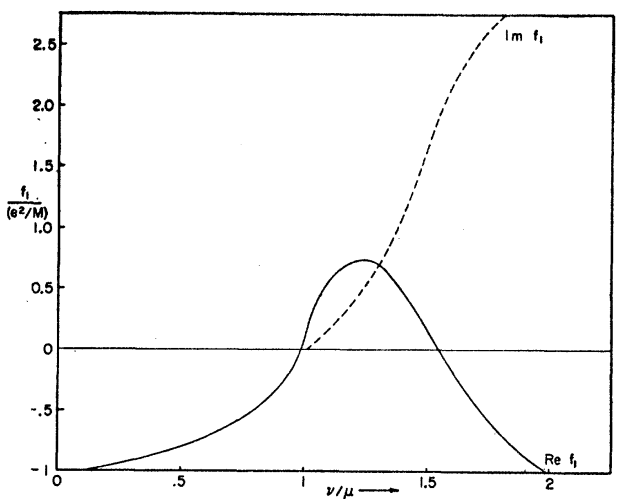

FIG. 2. The coherent forward scattering amplitude describing $\gamma$ rays scattered by protons as a function of photon energy (in units of meson mass).

where we have written $q=\left(\nu^{2}-1\right)^{\frac{1}{2}}$, taking the meson mass as unity. At low energies (up to $150 \mathrm{Mev}$ ) it seems quite reasonable to consider only dipole phase shifts. The photoproduction data can be interpreted as involving mainly $E_{\frac{1}{2}}{ }^{1}$ and $M_{\frac{3}{2}}{ }^{1}$ for $\pi^{+}$production and $M_{\frac{3}{2}}{ }^{1}$ for $\pi^{0}$ production. In choosing the numerical value of the phase shifts we have used the dispersion relation for $d_{1}(\nu)$, Eq. (5.14) to fix a particular sum of the phase shifts and find (for low frequencies):

$$
\begin{aligned}
& \frac{1}{\nu} \operatorname{Re}\left[\delta_{\frac{3}{2}} E_{1}+\delta_{\frac{3}{2}} M_{1}+\frac{1}{2}\left(\delta_{\frac{1}{2}} E_{1}+\delta_{\frac{1}{2}} M_{1}\right)\right] \\
& =-\frac{e^{2}}{M}+\frac{\nu^{2}}{2 \pi^{2}} \int_{\mu}^{\infty} d \nu^{\prime} \frac{\sigma\left(\nu^{\prime}\right)}{\nu^{\prime 2}}
\end{aligned}
$$

The sharing of the sum between $M_{\frac{3}{2}}{ }^{1}$ and $E_{\frac{1}{2}}{ }^{1}$ has been done in a way which seems in accordance with the photoproduction data. We have, however, made another assumption about the energy dependence of the anomalous part of the dipole phase shifts, and that is that they behave for small frequencies like $\nu^{3}$. From the Appendix and Eq. (5), one finds :

$$
\begin{aligned}
& \frac{1}{2 \nu} \operatorname{Re}\left[\delta_{\frac{3}{2}} E_{1}+\delta_{\frac{3}{2}} M_{1}-\right.\left.\left(\delta_{\frac{1}{2}} E_{1}+\delta_{\frac{1}{2}} M_{1}\right)\right] \\
&=\nu f_{2}^{\prime}(0)+\frac{2 \nu^{3}}{\pi} \int_{\mu}^{\infty} d \nu^{\prime} \frac{a_{2}\left(\nu^{\prime}\right)}{\nu^{\prime 4}}
\end{aligned}
$$

so that for that particular combination of phase shifts in Eq. (5.17) there may be a $\nu^{2}$ frequency dependence. [A physical model which yields for $f_{2}(\nu)$ such a linear frequency dependence is a particle with a Pauli-type anomalous magnetic moment.] There appears to be no general principle to guide one at this point. [Note added in proof.-This statement is incorrect. It has been shown by two of us (M. G-M. and M.L.G.) and inde- 


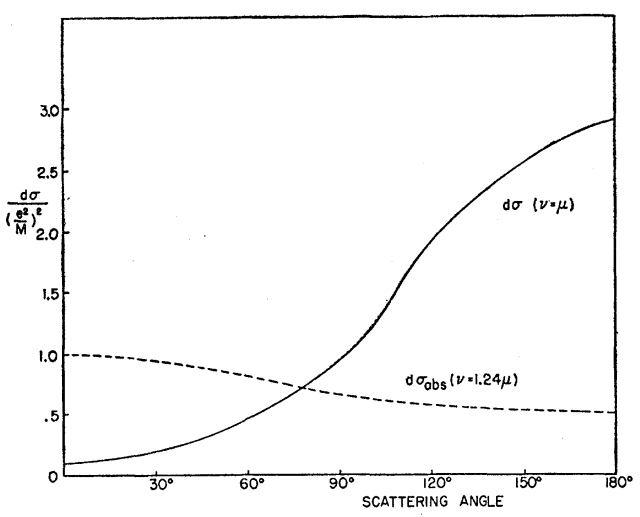

FIG. 3. The angular distribution of $\gamma$ rays scattered by protons computed on the basis of phase shifts given in Sec. V.

pendently by F. E. Low that the linear term in $f_{2}(\nu)$ arising from the anomalous magnetic moment is exactly correct in any known theory. This term does not appear in a lowest-order calculation in meson theory, since it is proportional to the square of the anomalous magnetic moment. The formula is $f_{2}^{\prime}(0)=-2\left(\mu_{\text {anom }}\right)^{2}$.

The phase shifts suggested above must be corrected for this effect.] If one computes the forward scattering amplitude using pseudoscalar meson theory, charged mesons only, one finds ${ }^{24}$ that the amplitude $f_{2}(\nu)$ is proportional to $\nu^{3}$. This appears to be in contradiction to the work of Sachs and Foldy ${ }^{25}$ who find $f_{2}(\nu) \sim \nu$, but we have been informed by Professor Sachs that an error has been found in the original work and they now find an $\nu^{3}$ dependence.

In Figs. 3 and 4 we show the angular distribution and the energy dependence of the $\gamma-P$ cross section which results from our assumed phase shifts. The angular

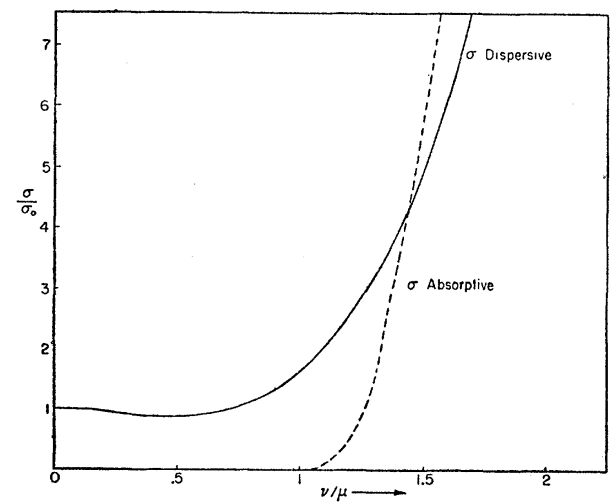

Fig. 4. The energy dependence of the total elastic scattering cross section for the scattering of $\gamma$ rays by protons. $\sigma_{0}$ is the Thomson cross section, $8 \pi e^{4} / 3 m^{2}$.

${ }^{24} \mathrm{M}$. L. Goldberger (unpublished).

${ }^{25}$ R. G. Sachs and L. Foldy, Phys. Rev. 80, 824 (1950). distribution is very sensitive to the ratio of $M_{\frac{3}{2}^{1}}$ and $E_{\frac{1}{2}}{ }^{1}$ phase shifts. The assumed dominance of $M_{\frac{3}{2}}{ }^{1}$ results in a strong backward maximum which should simplify the experimental verification of the effects, since largeangle scatterings would be most easily observed. The energy dependence of the total cross section is to be contrasted with that given by Sachs and Foldy. They found that the cross section fell sharply for frequencies greater than $\mu$, whereas our result shows a strong increase. Aside from the previously mentioned mistake in this paper, they inadvertantly dropped the absorptive part of the light-scattering amplitude. It should be emphasized that the results given in Figs. 3 and 4 are not to be taken too seriously, especially for energies above $1.5 \mu$. They are based on rather $a d$ hoc assumptions about phase shifts and are presented simply to give some qualitative ideas as to what one might expect.

We must finally discuss the omission of the Delbrück scattering. In the forward direction the scattering by the Coulomb field actually has a greater amplitude than the entire Compton amplitude considered thus far. One has $d_{1}$ (Delbrück) $\sim 10^{-15} \mathrm{~cm}$ as compared to $d_{1}($ Compton $) \sim 2 \times 10^{-16} \mathrm{~cm}$. The total cross section of the Delbrück scattering is, however, completely negligible $\left(\sim 10^{-34} \mathrm{~cm}^{2}\right)$. Furthermore, since the scattering is confined to very small angles, it should not affect the experimental search for anomalous $\gamma-P$ scattering.

We have been unable to find any useful applications of the dispersion relations for particles with mass; these can presumably be constructed, since as a function of the total energy, the scattering amplitude satisfies all the requirements imposed in Sec. IV. There are at least two obstacles in the way of such applications: (1) One needs, in general, the scattering amplitude for nonphysical energies, $\nu$, less than the mass, $\mu$. Unless one makes further assumptions, one cannot get a formula involving only measurable quantities. (2) There is no small parameter analogous to $e$ according to which one may separate the dispersive and absorptive effects. For example, an attempt to express the real part of the forward scattering amplitude for meson-nucleon scattering in terms of an integral over the total cross section [assuming point (1) is overcome] poses the problem that one knows neither side of the equation; the elastic scattering cross section is by no means negligible in comparison with the inelastic processes, as was the case for photons.

It is a pleasure to acknowledge several stimulating conversations with Professor E. P. Wigner. We are also indebted to Professor A. Wightman and to members of the Institute for Advanced Study for helpful conversations. One of us (W.T.) is grateful to the Institute for Advanced Study for a grant-in-aid and to Professor Robert Oppenheimer for the hospitality extended him at the Institute. 
APPENDIX

We wish to describe the scattering of photons by a spin one-half particle. It is convenient to use a description in which the incident photons are circularly polarized parallel or antiparallel to the spin of the nucleon. We have in deriving these formulas neglected the possibility that the electric and magnetic multipoles mix. (Stated otherwise, we have not used the eigenphases of the scattering matrix. ${ }^{26}$ The procedure is quite standard and we give only the result. The electric field vector $\mathbf{E}$ for the two cases of polarization becomes

$$
\begin{aligned}
\mathbf{E}_{p, a}= & -e^{i \nu z} \chi_{+} \alpha_{ \pm}+\frac{e^{i \nu r}}{r} \sum_{l=1}^{\infty}[2 \pi(2 l+1)]^{\frac{1}{2}} \\
& \times \sum_{j=l-\frac{1}{2}}^{j=l+\frac{1}{2}} C_{l_{\frac{1}{2}}}\left(j, 1 \pm \frac{1}{2} ; 1, \pm \frac{1}{2}\right) \\
& \times\left\{\frac{\exp \left(2 i \delta_{l j}{ }^{\mathrm{mag}}\right)-1}{2 i \nu}+i \frac{\exp \left(2 i \delta_{l j}{ }^{\mathrm{el}}\right)-1}{2 i \nu} \frac{\mathbf{r} \times}{r}\right\} \\
& \times \sum_{m=-\frac{1}{2}}^{m=\frac{1}{2}} C_{l_{2}}\left(j, 1 \pm \frac{1}{2} ; 1 \pm \frac{1}{2}-m, m\right) \\
& \times \mathbf{X}_{l, 1_{ \pm} \frac{1}{2}-m} \alpha_{m} .
\end{aligned}
$$

The notation is that of Blatt and Weisskopf $; 27$ the $\alpha_{ \pm}$ are the nucleon spin eigenfunctions. For the cross section, we obtain

$$
\begin{gathered}
d \sigma_{p}=2 \pi\left\{\mid \sum_{l=1}^{\infty}(2 l+1)^{\frac{1}{2}} \sum_{j=l-\frac{1}{2}}^{l+\frac{1}{2}}\left(\frac{\exp \left(2 i \delta_{l j} \mathrm{mag}\right)-1}{2 i \nu}\right.\right. \\
\left.+i \frac{\exp \left(2 i \delta_{l^{\prime}}{ }^{\mathrm{e}}\right)-1}{2 i \nu} \mathbf{r} \times\right)\left.\mathbf{X}_{l, 1}\left|C_{l \frac{1}{3}}\left(j, \frac{3}{2} ; 1, \frac{1}{2}\right)\right|^{2}\right|^{2} \\
+\mid \sum_{l=1}^{\infty}(2 l+1)^{\frac{1}{2}} \sum_{j=l-\frac{1}{2}}^{l+\frac{1}{2}}\left(\frac{\exp \left(2 i \delta_{l j} \mathrm{mag}\right)-1}{2 i \nu}\right. \\
\left.+\frac{\exp \left(2 i \delta_{l j}{ }^{\mathrm{el}}\right)-1}{2 i \nu} \mathbf{r} \times\right) \mathbf{X}_{l, 2} C_{l_{\frac{1}{2}}}\left(j, \frac{3}{2} ; 1, \frac{1}{2}\right) \\
\left.\times\left. C_{l_{\frac{1}{2}}}\left(j, \frac{3}{2} ; 2,-\frac{1}{2}\right)\right|^{2}\right\},
\end{gathered}
$$

${ }^{26} \mathrm{~J}$. Blatt and L. Biedenharn, Revs. Modern Phys. 23, 729 (1953)

${ }_{27}^{27}$ J. M. Blatt and V. F. Weisskopf, Theoretical Nuclear Physics (John Wiley and Sons, Inc., New York, 1952), Appendexes A and B.

$$
\begin{aligned}
& d \sigma_{a}=2 \pi\left\{\mid \sum_{l=1}^{\infty}(2 l+1)^{\frac{1}{2}} \sum_{j=l-\frac{1}{2}}^{l+\frac{1}{2}}\left(\frac{\exp \left(2 i \delta_{l j} \mathrm{mag}\right)-1}{2 i \nu}\right.\right. \\
&\left.+i \frac{\exp \left(2 i \delta_{l j}{ }^{\mathrm{e}}\right)-1}{2 i \nu} \mathbf{r} \times\right)\left.\mathbf{X}_{l, 1}\left|C_{l \frac{1}{2}}\left(j, \frac{1}{2} ; 1,-\frac{1}{2}\right)\right|^{2}\right|^{2} \\
&+\mid \sum_{l=1}^{\infty}(2 l+1)^{\frac{1}{2}} \sum_{j=l-\frac{1}{2}}^{l+\frac{1}{2}}\left(\frac{\exp \left(2 i \delta_{l j}{ }^{\mathrm{mag}}\right)-1}{2 i \nu}\right. \\
&\left.=i \frac{\exp \left(2 i \delta_{l j}{ }^{\mathrm{el}}\right)-1}{2 i \nu} \mathbf{r} \times\right) \mathbf{X}_{l, 0} C_{l \frac{1}{2}}\left(j, \frac{1}{2} ; 1,-\frac{1}{2}\right) \\
&\left.\times\left. C_{l \frac{1}{2}}\left(j, \frac{1}{2} ; 0, \frac{1}{2}\right)\right|^{2}\right\}
\end{aligned}
$$

In (B.1) the first and second term correspond to the coherent and spin-flip amplitudes, respectively. In the forward direction we obtain for the scattering amplitudes $f_{p}$ and $f_{a}$ the values

$$
\begin{aligned}
& f_{p}=\frac{3}{2 \nu}\left(\delta_{\frac{3}{2}} M_{1}+\delta_{\frac{3}{2}} E_{1}\right), \\
& f_{a}=\frac{1}{2 \nu}\left(\delta_{\frac{3}{2}} E_{1}+\delta_{\frac{3}{2}} M_{1}\right)+\frac{1}{\nu}\left(\delta_{\frac{1}{2}} E_{1}+\delta_{\frac{1}{2}} M_{1}\right) .
\end{aligned}
$$

We have assumed that the phase shifts are small and that only dipole radiation need be considered. In deriving (B.3), we have used the fact that the spin-flip amplitude vanishes in the forward direction and that

$$
\begin{aligned}
& \left.\mathbf{X}_{l, \pm 1}\right|_{\vartheta=0}= \pm \frac{1}{\sqrt{2}}\left(\frac{2 l+1}{4 \pi}\right)^{\frac{1}{2}} x_{ \pm} \\
& \underset{r}{\mathbf{r}} \times\left.\mathbf{X}_{l, \pm 1}\right|_{\vartheta=0}=\frac{1}{\sqrt{2}}\left(\frac{2 l+1}{4 \pi}\right)^{\frac{1}{2}} x_{ \pm} .
\end{aligned}
$$

In our application we assumed also that $\delta_{\frac{1}{2}} M_{1}=0$. We find for the cross section for unpolarized light:

$$
\begin{gathered}
d \sigma=\frac{1}{8 \nu^{2}}\left[\left(7+3 \cos ^{2} \vartheta\right)\left(\left|\delta_{\frac{3}{2}} M_{1}\right|^{2}+\left|\delta_{\frac{3}{2}} E_{1}\right|^{2}\right)\right. \\
+4\left|\delta_{\frac{1}{2}} E_{1}\right|^{2}+2 \operatorname{Re} \delta_{\frac{1}{2}} E_{1} \delta_{\frac{3}{2}} E_{1^{*}}{ }^{*}\left(3 \cos ^{2} \vartheta-1\right) \\
\left.+4 \operatorname{Re} \delta_{\frac{3}{2}}^{M_{1}} \delta_{\frac{3}{2}} E_{1} *(5+\cos \vartheta)\right], \\
\sigma=\frac{6 \pi}{\nu^{2}}\left\{\frac{2}{3}\left(\left|\delta_{\frac{3}{2}} M_{1}\right|^{2}+\left|\delta_{\frac{3}{2}} E_{1}\right|^{2}\right)+\frac{1}{3}\left|\delta_{\frac{1}{2}} E_{1}\right|^{2}\right\} .
\end{gathered}
$$

In deducing (B.4) we used Eq. XII, (3.16) of reference 28 and

$$
\mathbf{X}_{11} * \underset{r}{\underset{r}{\mathbf{r}}} \times \mathbf{X}_{11}=\frac{3}{8 \pi} \cos \vartheta, \quad \mathbf{X}_{10} * \underset{r}{i-} \times \mathbf{X}_{10}=0 .
$$

